\title{
ANALYSIS OF ITERATIVE METHODS FOR THE STEADY AND UNSTEADY STOKES PROBLEM: APPLICATION TO SPECTRAL ELEMENT DISCRETIZATIONS*
}

\author{
YVON MADAY†§, DAN MEIRON $\ddagger$, ANTHONY T. PATERA§, AND EINAR M. RØNQUIST§ণ
}

\begin{abstract}
A new and detailed analysis of the basic Uzawa algorithm for decoupling of the pressure and the velocity in the steady and unsteady Stokes operator is presented. The paper focuses on the following new aspects: explicit construction of the Uzawa pressure-operator spectrum for a semiperiodic model problem; general relationship of the convergence rate of the Uzawa procedure to classical inf-sup discretization analysis; and application of the method to high-order variational discretization.
\end{abstract}

Key words. Stokes problem, Uzawa decoupling, iterative methods, inf-sup, spectral elements

AMS(MOS) subject classifications. $65 \mathrm{I} 10,65 \mathrm{M} 70,65 \mathrm{~N} 12,65 \mathrm{~N} 25$

1. Introduction. The Stokes equations describe the motion of incompressible viscous fluid flow at very low Reynolds numbers. However, the need to have efficient Stokes solvers is not only limited to inertia free flows, but is also of great importance when solving numerically the full time-dependent Navier-Stokes equations. For moderate Reynolds numbers the nonlinear convective term is often treated explicitly, while the linear (Stokes) part is treated implicitly. In order for this semi-implicit approach to be attractive, efficient unsteady Stokes solvers are required.

Numerous approaches have been proposed for solving the algebraic system of equations resulting from discretization of the steady and unsteady Stokes equations. One approach is to solve the momentum and continuity equations directly in coupled form (e.g., Yamaguchi, Chang, and Brown [45] and Bathe and Dong [4]). This direct approach is general and robust; however, it can be inefficient and memory intensive for large, three-dimensional problems, in particular, for high-order methods. A second approach is to replace the discrete continuity equations with a Poisson equation for the pressure (e.g., Chorin [15], Temam [41], Glowinski and Pironneau [23], Kleiser and Schumann [27], Kim and Moin [26], and Orszag, Israeli, and Deville [35]). This approach decouples the momentum and continuity except on the domain boundary; however, it may require a rediscretization of the continuous problem, and boundary conditions must be supplied for the pressure.

A third approach, which we study more closely in this paper, is to apply a global nested iterative decoupling procedure for the pressure and the velocity. This scheme is an extension of the classical Uzawa algorithm (see Arrow, Hurwicz, and Uzawa [1], Chorin [15], Temam [41], Glowinski [22], and Girault and Raviart [21] for more basic concepts; see Cahouet and Chabard [13], Maday, Patera, and Rønquist [30], Fischer, Rønquist, Dewey, and Patera [19], Bristeau, Glowinski, and Periaux [12], Maday and Patera [31], and Cahouet and Chabard [14] for more recent advances). The Uzawa

* Received by the editors September 25, 1990; accepted for publication (in revised form) March 19, 1992. This work was supported by the Office of Naval Research and Defense Advanced Research Projects Agency contract N00014-89-J-1610, by Office of Naval Research contract N000114-88-K-0188, by National Science Foundation grant ASC-8806925, by Department of Energy Office of Energy Sciences grant DE-AS0376ER-72012, Applied Mathematical Sciences (KC-07-01-01), and by Nektonics Research Corporation.

$\dagger$ Université de Paris XII, Laboratoire d'Analyse Numérique de l'Université Pierre et Marie Curie, Paris, France.

¥California Institute of Technology, Applied Mathematics, 217-50, Pasadena, California 91125.

$\S$ Massachusetts Institute of Technology, Room 3-264, 77 Massachusetts Avenue, Cambridge, Massachusetts 02139 .

If Present address, Nektonics, 875 Main St., Cambridge, Massachusetts 02139. 
approach has several attractive features: It is more efficient in terms of computational complexity and memory requirement than a direct approach; it requires no pressure boundary conditions and no rediscretization of the original problem, and hence the convergence proofs for the original problem directly apply. In essence, by using a block Gaussian elimination procedure, this algorithm decouples the original saddle problem into two positive-semidefinite symmetric forms, one for the pressure and one for the velocity. Thus standard iterative procedures such as preconditioned conjugate gradient iteration and multigrid techniques can readily be applied.

In this paper we give a new and detailed analysis of the basic Uzawa algorithm. The paper focuses on the following new aspects: the explicit construction of the Uzawa pressure-operator spectrum for a particular case; the general relationship of the convergence rate of the Uzawa procedure to classical inf-sup discretization analysis [11], [3], and application of the method to high-order variational discretization. The outline of this paper is as follows. We start in $\S 2$ by reviewing the basic discretization of the steady and unsteady Stokes equations based on the equivalent variational forms. In $\S 3.1$ we review the Uzawa method for the steady Stokes problem, and in $\S 3.2$ we consider the full Fourier case. In $\S 3.3$ we proceed by presenting a new continuous analysis for a semiperiodic model problem. The analytical results regarding the good conditioning of the steady Stokes pressure operator are then verified numerically for optimal high-order spectral element discretizations. In $\S 3.4$ we discuss how these results extend to multidimensional spectral element discretizations, and present examples of steady Stokes problems solved by a nested preconditioned conjugate gradient/multigrid iteration scheme. Last, in $\S 4$ we analyze the Uzawa algorithm in the context of solving the unsteady Stokes equations.

2. The Stokes problems.

2.1. Steady Stokes. In this section we consider the steady Stokes problem in $d$ space dimensions: Find a velocity $\mathbf{u}$ and a pressure $p$ in a domain $\Omega \in \mathscr{R}^{d}$ such that

$$
\begin{aligned}
-\mu \Delta \mathbf{u}+\nabla p=\mathbf{f} & \text { in } \Omega, \\
-\nabla \cdot \mathbf{u}=0 & \text { in } \Omega,
\end{aligned}
$$

subject to homogeneous Dirichlet velocity boundary conditions on the domain boundary $\partial \Omega$,

$$
\mathbf{u}=\mathbf{0} \text { on } \partial \Omega \text {. }
$$

Here $\mathrm{f}$ is the prescribed force and $\mu$ is the viscosity. As mentioned in the Introduction, the solution to the Stokes problem (1), (2) is of interest, not only in its own right, but also in that it constitutes the major building block in many Navier-Stokes solvers. In this case, $f$ can be viewed as an augmented force which includes the explicitly treated nonlinear convective contributions.

The equivalent variational formulation of (1), (2) is: Find $(\mathbf{u}, p)$ in $X \times M$ such that

$$
\begin{aligned}
\mu(\nabla \mathbf{u}, \nabla \mathbf{w})-(p, \nabla \cdot \mathbf{w}) & =(\mathbf{f}, \mathbf{w}) \quad \forall \mathbf{w} \in X, \\
-(\nabla \cdot \mathbf{u}, q) & =0 \quad \forall q \in M,
\end{aligned}
$$

where the proper spaces for $\mathbf{u}$ and $p$ such that (4), (5) is well posed are [11], [21]

$$
\begin{aligned}
& X=\mathscr{H}_{0}^{1}(\Omega), \\
& M=\mathscr{L}_{0}^{2}(\Omega)=\mathscr{L}^{2}(\Omega) \cap\left\{\phi \in \mathscr{L}^{2}(\Omega) ; \int_{\Omega} \phi d \Omega=0\right\} .
\end{aligned}
$$


Here $\mathscr{L}_{0}^{2}(\Omega)$ is the space of all functions that are square integrable over $\Omega$ with zero average, while $\mathscr{H}_{0}^{1}(\Omega)$ is the space of all functions that are square integrable, whose derivatives are also square integrable over $\Omega$, and which satisfy the homogeneous boundary conditions (3).

Here we shall consider numerical approximations to the Stokes problem based on the variational form (4), (5): Find $\left(\mathbf{u}_{h}, p_{h}\right) \in\left(X_{h}, M_{h}\right)$ such that

$$
\begin{aligned}
\mu\left(\left(\nabla \mathbf{u}_{h}, \nabla \mathbf{w}\right)\right)_{h}-\left(p_{h}, \nabla \cdot \mathbf{w}\right)_{h} & =((\mathbf{f}, \mathbf{w}))_{h} \quad \forall \mathbf{w} \in X_{h}, \\
-\left(\nabla \cdot \mathbf{u}_{h}, q\right)_{h} & =0 \quad \forall q \in M_{h},
\end{aligned}
$$

where for each value of the parameter $h, X_{h} \subset X$ and $M_{h} \subset M$ are compatible subspaces of $X$ and $M$ (see [11], [3], [21], and [9]) that approach $X$ and $M$ as the discretization parameter $h$ goes to zero. In $(8),(9)(\cdot, \cdot)_{h}$ and $((\cdot, \cdot))_{h}$ denote evaluation of the continuous inner product $(\cdot, \cdot)$ by Gauss numerical quadrature (note, however, that the $(\cdot, \cdot)_{h}$ and $((\cdot, \cdot))_{h}$ may be different $)$.

Choosing appropriate (compatible) discrete spaces $X_{h}$ and $M_{h}$ with associated bases, we arrive at a set of algebraic equations given in matrix form as

$$
\begin{aligned}
\mu \underline{A} \underline{u}_{i}-\underline{D}_{i}^{T} \underline{p} & =\underline{B} \underline{f}_{i}, \quad i=1, \ldots, d, \\
-\underline{D}_{i} \underline{u}_{i} & =0,
\end{aligned}
$$

where $\underline{A}$ is the discrete Laplace operator, $\underline{B}$ is the mass matrix, $\underline{D}^{T}=\left(\underline{D}_{1}^{T}, \ldots, \underline{D}_{d}^{T}\right)$ is the discrete gradient operator, and the underscore refers to basis coefficients. In (10), (11), we assume that the homogeneous boundary conditions are imposed by eliminating appropriate rows and columns. Note that in the limit as the discretization parameter $h \Rightarrow 0,\left(X_{h}, M_{h}\right) \Rightarrow(X, M)$, and (10), (11) applies even for the continuous case.

2.2. Unsteady Stokes. The unsteady Stokes equations are given by

$$
\begin{aligned}
-\mu \Delta \mathbf{u}+\nabla p+\rho \frac{\partial \mathbf{u}}{\partial t}=\mathbf{f} & \text { in } \Omega, \\
-\nabla \cdot \mathbf{u}=0 & \text { in } \Omega,
\end{aligned}
$$

with boundary and initial conditions

$$
\begin{aligned}
\mathbf{u} & =\mathbf{0} & & \text { on } \partial \Omega, \\
\mathbf{u}(\mathbf{x}, t=0) & =\mathbf{g}(\mathbf{x}) & & \text { in } \Omega .
\end{aligned}
$$

Here all variables are defined as in the steady case with $t$ representing time, and $\rho$ the density of the fluid. Although there are physical problems in which the unsteady Stokes equations are relevant, the unsteady problem is primarily of interest with regard to its role in unsteady Navier-Stokes calculations.

We proceed directly to the time discretization of (12), (13) by an implicit Euler backward method (readily extended to Crank-Nicolson)

$$
\begin{aligned}
-\mu \Delta \mathbf{u}^{n+1}+\nabla p^{n+1}+\rho \frac{\mathbf{u}^{n+1}-\mathbf{u}^{n}}{\Delta t} & =\mathbf{f}^{n+1}, \\
-\nabla \cdot \mathbf{u}^{n+1} & =0,
\end{aligned}
$$

in which $\left(\mathbf{u}^{n}, p^{n}\right)$ represents an approximation of $(\mathbf{u}(\mathbf{x}, n \Delta t), p(\mathbf{x}, n \Delta t))$, and $\Delta t$ is the time step. 
The spatial discretization of (16), (17) follows the same procedure as for the steady case. At each time step we search for a discrete solution $\left(\mathbf{u}_{h}^{n+1}, p_{h}^{n+1}\right)$ in the finitedimensional (compatible) subspaces $X_{h} \subset X$ and $M_{h} \subset M$, and we arrive at a set of algebraic equations to be solved for the nodal values $\underline{u}^{n+1}=\left(\underline{u}_{1}^{n+1}, \ldots, \underline{u}_{d}^{n+1}\right)$ and $\underline{p}^{n+1}$,

$$
\begin{aligned}
\mu \underline{A} \underline{u}_{i}^{n+1}-\underline{D}_{i}^{T} \underline{p}^{n+1}+\rho \underline{B}\left(\frac{\underline{u}_{i}^{n+1}-\underline{u}_{i}^{n}}{\Delta t}\right) & =\underline{B}_{i}^{n+1}, \quad i=1, \ldots, d, \\
-\underline{D}_{i} \underline{u}_{i}^{n+1} & =0 .
\end{aligned}
$$

We note that for any discretization (18), (19) for which $\underline{A}$ is positive-definite symmetric, (18), (19) is unconditionally stable $(\mathbf{f}=\mathbf{0})$,

$$
\left\|\mathbf{u}_{h}^{n+1}\right\|_{0, h}<\left\|\mathbf{u}_{h}^{n}\right\|_{0, h},
$$

as can be readily demonstrated by multiplying (18) and (19) by $\underline{u}_{i}^{n+1}$ and $\underline{p}^{n+1}$, respectively. In (20) $\|\cdot\|_{0, h}$ denotes the discrete $\mathscr{L}^{2}$-norm, $\|v\|_{0, h}=((v, v))_{h}^{1 / 2}$.

\section{Steady Stokes solvers.}

3.1. The Uzawa algorithm. The classical Uzawa scheme originates from economic theory as a saddle-point approach to solving constrained optimization problems (see Arrow, Hurwicz, and Uzawa [1]). Following Brezzi [11] and Girault and Raviart [21], the Stokes problem (4), (5) can be formulated as the following equivalent saddle-point problem: Find a pair $(\mathbf{u}, p) \in X \times M$ such that

$$
\mathscr{T}(\mathbf{u}, q) \leqq \mathscr{T}(\mathbf{u}, p) \leqq \mathscr{T}(\mathbf{v}, p) \quad \forall \mathbf{v} \in X, \quad \forall q \in M,
$$

where the quadratic Lagrangian functional $\mathscr{T}: X \times M \rightarrow \mathscr{R}$ is defined by

$$
\mathscr{T}(\mathbf{v}, q)=\frac{\mu}{2}(\nabla \mathbf{v}, \nabla \mathbf{v})-(\mathbf{f}, \mathbf{v})-(q, \nabla \cdot \mathbf{v}) .
$$

The constraint in the Stokes problem is the incompressibility condition, while pressure plays the role of the Lagrange multiplier. In the case of finding a numerical approximation to the Stokes problem (1)-(3), the equivalence between the discrete formulation (8), (9) and a finite-dimensional saddle-point problem is now readily seen: Find $\left(\mathbf{u}_{h}, p_{h}\right) \in X_{h} \times M_{h}$ such that

$$
\mathscr{T}_{h}\left(\mathbf{u}_{h}, q\right) \leqq \mathscr{T}_{h}\left(\mathbf{u}_{h}, p_{h}\right) \leqq \mathscr{T}_{h}\left(\mathbf{v}, p_{h}\right) \quad \forall \mathbf{v} \in X_{h}, \quad \forall q \in M_{h},
$$

where the quadratic Lagrangian functional $\mathscr{T}_{h}: X_{h} \times M_{h} \rightarrow \mathscr{R}$ is defined by

$$
\mathscr{T}_{h}(\mathbf{v}, q)=\frac{\mu}{2}((\nabla \mathbf{v}, \nabla \mathbf{v}))_{h}-((\mathbf{f}, \mathbf{v}))_{h}-(q, \nabla \cdot \mathbf{v})_{h} .
$$

In terms of finding the nodal values $\underline{\underline{u}}$ and $p$ in (10), (11), the classical Uzawa approach to solving the min-max problem (23) is characterized by the following gradient method [41]:

$$
\begin{aligned}
\mu \underline{A} \underline{u}_{i}^{m+1} & =\underline{D}_{i}^{T} \underline{p}^{m}+\underline{B} \underline{f}_{i}, \quad i=1, \ldots, d ; \\
\underline{\tilde{B}} \underline{p}^{m+1} & =\underline{\tilde{B}} \underline{p}^{m}-\alpha \underline{D}_{i} \underline{\mathbf{u}}_{i}^{m+1} .
\end{aligned}
$$

Here $m$ is the iteration counter, $\alpha \in \mathscr{R}$ is a positive iteration parameter, and $\underline{\tilde{B}}$ is the mass matrix associated with the bilinear form $(\phi, \psi)_{h}$ for all $\phi, \psi \in M_{h}$. In (25) we minimize $\mathscr{T}_{h}\left(\mathbf{v}, p_{h}^{m}\right)$ for all $\mathbf{v} \in X_{h}$, while in (26) we try to maximize $\mathscr{T}_{h}\left(\mathbf{u}_{h}^{m}, q\right)$ for all $q \in M_{h}$. For sufficiently small $\alpha$, the two-level iteration scheme (25), (26) converges to the solution of (10), (11). 
As is the case for many gradient algorithms, the Uzawa procedure in the form (25), (26) converges very slowly, especially for large multidimensional problems. The convergence rate can be improved by considering augmented Lagrangian methods (Fortin and Glowinski [20]), or multigrid schemes (Verfurth [44] and Maître, Musy, and Nigon [34]). However, replacing (25), (26) by conjugate gradient iteration can also accelerate the convergence significantly to give very good results. For details in the finite-element context, we refer to Glowinski [22] and Girault and Raviart [21]. In the following we shall demonstrate, both in terms of continuous analysis and numerical examples, that the latter approach is very attractive in terms of conditioning, computational complexity, and parallelism.

We begin with a decoupling of the original saddle problem (10), (11) into two positive (semi)definite symmetric forms, one for the velocity and one for the pressure. First, for each of the velocity components $\underline{u}_{i}$ from the momentum equations (10), we formally solve

$$
\underline{u}_{i}=\underline{A}^{-1} \underline{D}_{i}^{T} \underline{p}+\underline{A}^{-1} \underline{B} \underline{f}_{i}, \quad i=1, \ldots, d .
$$

We then insert (27) into the continuity equation (11) to arrive at

$$
0=-\underline{D}_{i} \underline{u}_{i}=-\underline{D}_{i} \underline{A}^{-1} \underline{D}_{i}^{T} \underline{p}-\underline{D}_{i} \underline{A}^{-1} \underline{B} \underline{f}_{i} .
$$

Thus the discrete saddle problem (10), (11) can be replaced with the discretely equivalent statement

$$
\begin{aligned}
\underline{A} \underline{u}_{i}-\underline{D}_{i}^{T} \underline{p} & =\underline{B} \underline{f}_{i}, \\
\underline{S} \underline{p} & =-\underline{D}_{i} \underline{A}^{-1} \underline{B} \underline{f}_{i},
\end{aligned}
$$

where the discrete pressure operator

$$
\underline{S}=\underline{D}_{i} \underline{A}^{-1} \underline{D}_{i}^{T}
$$

is a positive-semidefinite symmetric matrix. Hence, the saddle problem (10), (11) can be solved by first maximizing $\mathscr{T}_{h}\left(\mathbf{u}_{h}, q\right)$ for all $q \in M_{h}$ (see (30)), and then minimizing $\mathscr{T}_{h}\left(\mathbf{v}, p_{h}\right)$ for all $\mathbf{v} \in X_{h}$ (see (29)).

We now make several comments regarding the system (29), (30). First, we note that the equation set (29), (30) does not correspond to a rediscretization of the continuous problem, that is, (29), (30) is equivalent to (10), (11). This implies that the theoretical error estimates derived for (8), (9) directly apply (in the case of spectral element discretizations, we refer to Maday and Patera [31] and Maday, Patera, and Rønquist [30]). Second, since the system matrices $\underline{S}$ and $\underline{A}$ are symmetric positive (semi)definite, standard elliptic solvers such as conjugate gradient iteration or multigrid techniques can readily be applied. The system (29), (30) is solved by first solving (30) for the pressure $\underline{p}$ and then solving (29) for the velocity $\underline{u}_{i}, i=1, \ldots, d$ with $\underline{p}$ known. Third, the pressure-operator $\underline{S}$ is completely full due to the embedded inverse $\underline{A}^{-1}$, and thus clearly necessitates an iterative approach.

Heuristically we expect the continuous pressure-operator $s$ to be close to the identity operator $I$ and therefore to be well conditioned. To see this, we formally apply the Uzawa decoupling procedure to the continuous equations (1), (2) and neglect boundary conditions

$$
s \sim \nabla \cdot(\Delta)^{-1} \nabla \sim I
$$

In the discrete case we do not expect $\underline{S}$ to be close to the identity matrix $\underline{I}$, but rather the variational equivalent of the identity operator, the mass matrix $\underline{\tilde{B}}$. Hence, we expect that

$$
\underline{\tilde{B}}^{-1} \underline{S} \sim \underline{I}
$$


suggesting that we can invert $\underline{S}$ efficiently by conjugate gradient iteration, using the mass matrix $\underline{\tilde{B}}$ as a preconditioner. Note here the importance of the proper choice of bases and numerical quadratures in order to define a matrix $\underline{\tilde{B}}$ that is easy to invert, that is, in order for $\underline{\tilde{B}}$ to be diagonal.

The preconditioned conjugate gradient iteration (outer iteration) for the system (30) takes the form [24], [21],

$$
\begin{gathered}
\underline{p}_{0} ; \quad \underline{r}_{0}=\underline{D}_{i} \underline{A}^{-1} \underline{B}_{i}+\underline{S} \underline{p}_{0} ; \quad \underline{\psi}_{0}=\underline{\tilde{B}}^{-1} \underline{r}_{0} ; \quad \underline{\phi}_{0}=\underline{\psi}_{0} ; \\
a_{m}=-\frac{\underline{\psi} \underline{\underline{r}}_{m}}{\underline{\phi}_{m}^{T} \underline{S} \underline{\phi}_{m}}, \quad \underline{p}_{m+1}=\underline{p}_{m}+a_{m} \underline{\phi}_{m}, \quad \underline{r}_{m+1}=\underline{r}_{m}+a_{m} \underline{S} \underline{\phi}_{m}, \\
\underline{\psi}_{m+1}=\underline{\tilde{B}}^{-1} \underline{\underline{r}}_{m+1}, \quad b_{m}=\frac{\underline{\psi}_{m+1}^{T} \underline{r}_{m+1}}{\underline{\psi}_{m}^{T} \underline{\underline{r}}_{m}}, \quad \underline{\phi}_{m+1}=\underline{r}_{m+1}+b_{m} \underline{\phi}_{m},
\end{gathered}
$$

where $m$ refers to the iteration number, $\underline{r}_{m}$ is the residual, $\underline{\phi}_{m}$ is the search direction, $\tilde{B}$ is the preconditioner, $\underline{\psi}_{m}$ is a vector associated with the preconditioning, and $a_{m}$ and $b_{m}$ are scalars.

The inner iteration is associated with the evaluation of the matrix-vector product $\underline{S} \phi$ in the outer conjugate gradient iteration. From the definition of $\underline{S}$ in (31) this evaluation can be performed as follows:

$$
\begin{aligned}
\underline{y}_{i} & =\underline{D}_{i}^{T} \underline{\phi} \quad i=1, \ldots, d, \\
\underline{A} \underline{z}_{i} & =\underline{y}_{i} \quad i=1, \ldots, d, \\
\underline{S} \underline{q} & =\underline{D}_{i} \underline{z}_{i} .
\end{aligned}
$$

We see that for general discretizations, each matrix-vector product evaluation requires $d$ standard elliptic Laplacian solves in $\mathscr{R}^{d}$. In order for this approach to be efficient for large multidimensional problems, the discrete Laplace operator $\underline{A}$ must be inverted by a fast solver, such as a good preconditioned conjugate gradient solver. In summary, the pressure is computed from (30) by effecting the nested inner/outer iteration procedure (34)-(37).

If the condition number of the matrix $\underline{\tilde{B}}^{-1} \underline{S}$ is order unity, we see that the above algorithm requires only order- $d$ elliptic solves, and hence represents an ideal decoupling of the Stokes problem. We also note that the residual $\underline{r}$ in the outer conjugate gradient iteration (34) is precisely the discrete divergence $-\underline{D}_{i} \underline{u}_{i}$. This is a useful result, as it allows for direct control of the discrete divergence when specifying the tolerance for the outer iteration. (The proper choice of tolerances in any nested iterative procedure is an important issue, and will be addressed separately in a future paper.)

We now make some general remarks regarding the relation between the inf-sup condition due to Babuska [3] and Brezzi [11], and the accuracy and efficiency by which the pressure can be computed. The necessary and sufficient condition for well-posedness of the saddle problem (8), (9) can be written as: there exists a real $\beta_{h}>0$ such that for all $q \in M_{h}$, there exists $v \in X_{h}$,

$$
\beta_{h}\|q\|_{0, h} \leqq \frac{(q, \nabla \cdot \mathbf{v})_{h}}{|\mathbf{v}|_{1, h}},
$$

where $\|\cdot\|_{0, h}$ is the discrete $\mathscr{L}^{2}$-norm associated with the pressure mesh $\left(M_{h}\right)$,

$$
\|q\|_{0, h}^{2}=(q, q)_{h}=\underline{q}^{T} \underline{\tilde{B}} q,
$$

and $|\cdot|_{1, h}$ is the discrete seminorm associated with the velocity mesh $\left(X_{h}\right)$,

$$
|\mathbf{v}|_{1, h}^{2}=\left(\left(\nabla v_{i}, \nabla v_{i}\right)\right)_{h}=\underline{v}_{i}^{T} \underline{A} \underline{v}_{i} \text {. }
$$


In (34) we suggest solving (30) by conjugate gradient iteration, using $\tilde{\tilde{B}}$ as a preconditioner. To estimate the efficiency of this approach it is of interest to determine the condition number $\kappa^{S}$ of the matrix $\underline{\tilde{B}}^{-1} \underline{S}$. For general discretizations it can be shown that the inf-sup parameter $\beta_{h}$ is closely related to the minimum eigenvalue of the pressure-operator $\underline{S}$ (see Appendix A)

$$
\beta_{h}^{2}=\lambda_{\min }^{S}
$$

where $\lambda_{\min }^{S}$ is the minimum eigenvalue of $\underline{S}$ with respect to the mass matrix $\underline{\tilde{B}}$,

$$
\lambda_{\min }^{S}=\min _{\underline{\phi}} \frac{\underline{\phi}^{T} \underline{S} \underline{\phi}}{\underline{\phi}^{T} \underline{\tilde{B}} \underline{\phi}} .
$$

It can also be shown (see Appendix A) that the maximum eigenvalue of $\underline{S}$ with respect to $\underline{\tilde{B}}, \lambda_{\max }^{S}$, is of order unity, implying that the condition number $\kappa^{S}$ is given as

$$
\kappa s=\frac{\lambda_{\max }^{S}}{\lambda_{\min }^{S}}=\frac{C}{\beta_{h}^{2}},
$$

where $C$ is a constant of order unity. Thus the number of outer conjugate gradient iterations scales like $1 / \beta_{h}$ [24]. If $\beta_{h}$ is of order unity, the outer iteration' (34) converges in order-one iterations.

The inf-sup parameter $\beta_{h}$ also affects the accuracy by which the pressure can be computed; in fact, it can be shown that the error in the pressure $p_{h}$ is inversely proportional to $\beta_{h}$, [11], [21], [10],

$$
\left\|p-p_{h}\right\|_{0} \leqq \frac{C}{\beta_{h}}\left(\inf _{q_{h} \in M_{h}}\left\|p-q_{h}\right\|_{0}+\cdots\right),
$$

where the dots indicate error terms originating from the velocity and forcing terms. However, the velocity remains unaffected by the inf-sup parameter

$$
\left\|\mathbf{u}-\mathbf{u}_{h}\right\|_{1} \leqq C\left(\inf _{\mathbf{v}_{h} \in X_{h, 0}}\left\|\mathbf{u}-\mathbf{v}_{h}\right\|_{1}+\cdots\right),
$$

where

$$
X_{h, 0}=\left\{\mathbf{v}_{h} \in X_{h} \mid\left(\nabla \cdot \mathbf{v}_{h}, q_{h}\right)_{h}=0 \forall q_{h} \in M_{h}\right\},
$$

thereby proving that the error in velocity is of the same size as the best fit by discrete divergence-free functions. Even though, in some cases, the presence of weakly spurious modes gives rise to poor approximation by $X_{h, 0}$, in many interesting cases we have

$$
\inf _{\mathbf{v}_{h} \in X_{h}}\left\|\mathbf{u}-\mathbf{v}_{h}\right\|_{1} \sim \inf _{\mathbf{v}_{h} \in X_{h, 0}}\left\|\mathbf{u}-\mathbf{v}_{h}\right\|_{1}
$$

[9], [10], [5], [39], [25].

For reasons of accuracy and efficiency we can now see that it is of great importance that $\beta_{h}$ be independent of the mesh parameter $h$. In most finite-element applications the inf-sup parameter is resolution-independent as long as the discrete spaces are compatible. However, in spectral methods this is not the case, and weakly spurious modes [42] are observed. These modes are responsible for an inf-sup parameter $\beta_{h}$ that depends on the mesh parameter $h \sim 1 / N$, where $N$ is the polynomial degree chosen for the approximation. For example, in the pure spectral case when $X_{h}$ and $M_{h}$ consist of all polynomials of degree $\leqq N$, and all (strong) spurious modes for the pressure are eliminated [9], there still exist weakly spurious modes responsible for an inf-sup parameter $\beta_{h} \sim \mathcal{O}(h) \sim \mathcal{O}\left(N^{-1}\right)$ [7], [43]. This has led to the construction of 
alternative methods based on staggered meshes in order to avoid strong spurious modes and to minimize the effects of weakly spurious modes. We refer to Bernardi and Maday [6], and $\S \S 3.3$ and 3.4 for a description of such methods.

The Uzawa algorithm is well known as an efficient way of solving the algebraic system of equations (10), (11) resulting from (low-order) finite-element discretization of the steady Stokes problem (1)-(3). The adaptation of the method to the spectral case is rather new, as described in Maday, Patera, and Rønquist [30], Streett, Hussaini, and Maday [40], Azarez, Labrosse, and Vandeven [2], and Lequéré [29]. In the following we investigate in detail the conditioning of the steady Stokes pressure operator; in particular we shall look at the full Fourier case, a semiperiodic model problem (both continuous and spectral element case), and the multidimensional spectral element case.

3.2. Full Fourier case. We start by first considering the simple case of Fourier discretization in $\mathscr{R}^{d}$, in which we choose the approximation spaces $X_{h}$ and $M_{h}$ to be

$$
\begin{gathered}
X_{h}=M_{h}^{3}, \\
M_{h}=\operatorname{span}\left\{e^{i \mathbf{k} \cdot \mathbf{x}},\left|k_{j}\right|<\mathscr{K}, \forall j=1, \ldots, d\right\}
\end{gathered}
$$

where $\mathbf{k}=\left(k_{1}, k_{2}, k_{3}\right)$ is the wave vector, $\mathbf{x}=\left(x_{1}, x_{2}, x_{3}\right) \in \Omega$, and $\mathscr{K}$ is the maximum wave number in each spatial direction. Reality is imposed by conjugate symmetry. For this Fourier discretization it is clear that

$$
\begin{gathered}
\underline{B} \Rightarrow 1, \\
\underline{D} \Rightarrow i k_{j}, \\
\underline{A} \Rightarrow-k^{2}=-\sum_{j=1}^{d} k_{j} k_{j},
\end{gathered}
$$

from which it follows that $\underline{S}=\underline{I}$ independent of $\mathscr{K}$. For the Fourier case the Uzawa algorithm is perfectly conditioned, as might be expected; see Maday and Quarteroni [33] for a numerical analysis of this spatial discretization.

\subsection{Semiperiodic case.}

3.3.1. Continuous case. Next, we turn to the analysis of the semiperiodic problem. This problem includes boundaries, and is thus much more instructive than the full Fourier case, yet it is sufficiently simple to allow for a complete analysis. The semiperiodic model problem corresponds to the domain $\Omega=]-1,1[\times] 0,2 \pi[$, with $(x, y)$ denoting a point in $\Omega$. The semiperiodic boundary conditions we consider are

$$
\begin{gathered}
\forall y \in] 0,2 \pi[, \quad \mathbf{u}(-1, y)=\mathbf{u}(1, y)=\mathbf{0}, \\
\forall x \in]-1,1[, \quad \mathbf{u}(x, 0)=\mathbf{u}(x, 2 \pi),
\end{gathered}
$$

and the associated spaces are

$$
\begin{aligned}
& X=\left\{v \in \mathscr{H}^{1}(\Omega) \mid v \text { satisfies }(53)-(54)\right\}, \\
& M=\mathscr{L}_{0}^{2}(\Omega),
\end{aligned}
$$

where $\mathscr{L}_{0}^{2}$ is defined in (7).

We now write the velocity, the pressure, and the data as a Fourier series in the periodic $y$-direction,

$$
\mathbf{u}(x, y)=\sum_{k=-\infty}^{\infty} \hat{\mathbf{u}}^{k}(x) e^{i k y}
$$




$$
\begin{aligned}
& p(x, y)=\sum_{k=-\infty}^{\infty} \hat{p}^{k}(x) e^{i k y}, \\
& \mathbf{f}(x, y)=\sum_{k=-\infty}^{\infty} \hat{\mathbf{f}}^{k}(x) e^{i k y}
\end{aligned}
$$

and use the orthogonality of the Fourier modes to reduce the steady Stokes problem to a series of decoupled (continuous) problems: Find $\hat{\mathbf{u}}^{k}=\left(\hat{u}^{k}, \hat{v}^{k}\right)$ and $\hat{p}^{k}$ in $\hat{X} \times \hat{M}$ such that

$$
\begin{aligned}
\hat{u}_{x x}^{k}-k^{2} \hat{u}^{k} & =\hat{p}_{x}^{k}+\hat{f}^{k}, \\
\hat{v}_{x x}^{k}-k^{2} \hat{v}^{k} & =i k \hat{p}^{k}+\hat{g}^{k}, \\
\hat{u}_{x}^{k}+i k \hat{v}^{k} & =0,
\end{aligned}
$$

where

$$
\begin{gathered}
\hat{X}=\mathscr{H}_{0}^{1}(\Lambda), \\
\hat{M}=\mathscr{L}_{0}^{2}(\Lambda),
\end{gathered}
$$

$\Lambda=]-1,1\left[\right.$, subscript $x$ denotes differentiation with respect to $x, \hat{\mathbf{f}}^{k}=\left(\hat{f}^{k}, \hat{g}^{k}\right)$, and we consider wave numbers $k \neq 0$.

From (60)-(62) we now readily derive the following expression for the continuous pressure operator $s^{k}$ acting on any $\hat{p}^{k}$ associated with wave number $k$ in the periodic $y$-direction

$$
s^{k}\left(\hat{p}^{k}\right)=\int_{-1}^{1}\left[\frac{\partial}{\partial x} G\left(x, x^{\prime}\right) \frac{\partial}{\partial x^{\prime}}-k^{2} G\left(x, x^{\prime}\right)\right] \hat{p}^{k}\left(x^{\prime}\right) d x^{\prime},
$$

where $G\left(x, x^{\prime}\right)$ is the Green's function for the second-order problem:

$$
\begin{aligned}
G_{x x}-k^{2} G & =\delta\left(x-x^{\prime}\right), \\
G\left(-1, x^{\prime}\right) & =G\left(1, x^{\prime}\right)=0 .
\end{aligned}
$$

The solution to (66), (67) can be expressed in closed form as

$$
G\left(x, x^{\prime}\right)=\frac{-1}{k \sinh 2 k} \sinh k\left(1+x^{<}\right) \sinh k\left(1-x^{>}\right),
$$

where

$$
\begin{aligned}
& x^{<}= \begin{cases}x & \text { for } x<x^{\prime}, \\
x^{\prime} & \text { for } x>x^{\prime},\end{cases} \\
& x^{>}= \begin{cases}x^{\prime} & \text { for } x<x^{\prime}, \\
x & \text { for } x>x^{\prime} .\end{cases}
\end{aligned}
$$

To find the condition number of $s^{k}$, we analyze the spectrum of the following Fredholm integral equation

$$
\int_{-1}^{1} \mathscr{G}\left(x, x^{\prime}\right) \chi\left(x^{\prime}\right) d x^{\prime}=\lambda^{s} \chi(x)
$$


where the kernel $\mathscr{G}$ follows from (65) as

$$
\mathscr{G}\left(x, x^{\prime}\right)=\frac{\partial}{\partial x} G\left(x, x^{\prime}\right) \frac{\partial}{\partial x^{\prime}}-k^{2} G\left(x, x^{\prime}\right)
$$

Substituting the expression (68) for the Green's function into (72), the solution to (71) can be found by inspection (see Appendix B). The entire spectrum of $s^{k}$ is given as

$$
\begin{aligned}
& \lambda_{1}^{s}(k)=\frac{1}{2}-\frac{k}{\sinh 2 k}, \\
& \lambda_{2}^{s}(k)=\frac{1}{2}+\frac{k}{\sinh 2 k}, \\
& \lambda_{l}^{s}(k)=1, \quad l>2,
\end{aligned}
$$

with only one nonunity eigenvalue for each boundary. The fact that there are only two nonunity $\lambda_{l}^{s}$ 's is related to the fact that for the Stokes problem, the pressure and velocity are only coupled at boundaries. (This can also be seen by taking the divergence of the momentum equation, which yields $\Delta p=\nabla \cdot f$ in $\Omega$, but indeterminacy at the boundaries. The proper boundary conditions are, in fact, $\nabla \cdot \mathbf{u}=0$.)

For a given wave number $k$, the condition number of $s^{k}$ is given by

$$
\kappa^{s}(k)=\left(\frac{1}{2}-\frac{k}{\sinh 2 k}\right)^{-1} .
$$

Since the spectrum (73)-(75) is clustered with only three distinct eigenvalues, the outer conjugate gradient iteration in the pressure solver will converge in three iterations independent of the condition number $\kappa^{s}(k)$. However, this result is only useful for semiperiodic discretizations; for truly multidimensional problems we must consider the condition number for all admissible $k$. In particular, if we all ow wave numbers in the range $1 \leqq k<k_{\max }$, we find that

$$
\kappa^{s}=\left(\frac{1}{2}-\frac{1}{\sinh 2}\right)^{-1} \cong 4.46,
$$

which does not depend on $k_{\max }$, and hence will not depend on the number of discrete degrees of freedom in the system.

3.3.2. Spectral element discretization. The above continuous analysis suggests that even in the presence of walls, the spectrum of the discrete pressure operator $\underline{S}$ with respect to the mass matrix $\underline{\tilde{B}}$ is clustered near unity, with a condition number that should be largely independent of the discretization parameter $h$. Here we are primarily interested in spectral element discretizations, corresponding to spaces $X_{h}$ and $\boldsymbol{M}_{h}$ consisting of piecewise high-order polynomials [36], [31], [37].

In order to construct the discrete pressure operator $\underline{S}$, the decoupled (continuous) equations (60)-(62) for each Fourier mode $k$ are discretized using spectral elements in the nonperiodic $x$-direction. The discretization procedure starts by breaking up the domain $\Lambda=$ ]-1, 1 [ into $K$ equal elements

$$
\bar{\Lambda}=\bigcup_{k=1}^{K} \bar{\Lambda}_{k}
$$

We then choose the subspaces to be

$$
\hat{X}_{h}=\hat{X} \cap \mathscr{P}_{N, K}(\Lambda),
$$




$$
\hat{M}_{h}=\hat{M} \cap \mathscr{P}_{N-2, K}(\Lambda),
$$

where

$$
\mathscr{P}_{n, K}(\Lambda)=\left\{\Phi \in \mathscr{L}^{2}(\Lambda) ;\left.\Phi\right|_{\Lambda_{k}} \in \mathscr{P}_{n}\left(\Lambda_{k}\right)\right\},
$$

and $\mathscr{P}_{n}\left(\Lambda_{k}\right)$ denotes the space of all polynomials of degree less than or equal to $n$ with respect to $x$. The discretization parameter $h$ is thus characterized by two numbers, the number of elements $K$, and the polynomial degree within each element $N$. In the following, we shall use the notation $h=(K, N)$. We refer to Maday, Patera, and Rønquist [30] and Bernardi, Maday, and Métivet [10] for a justification of the choice of discrete spaces.

The velocity and pressure are now expressed in terms of high-order Lagrangian interpolant bases through the Gauss-Lobatto and Gauss points, respectively [31]. This choice of bases results in minimal interelemental couplings, while still preserving the required $C^{0}$-continuity of the velocity across elemental boundaries. The inner products in (8), (9) are evaluated using Gauss numerical quadrature [16], Gauss-Legendre for $(\cdot, \cdot)_{h}$, and Gauss-Lobatto-Legendre for $((\cdot, \cdot))_{h}$. Choosing appropriate test functions, we arrive at a set of algebraic equations of the form (10), (11), which are then decoupled into the form (29), (30). Note that for Legendre spectral element discretizations, the quadrature points are the same as the collocation points, resulting in diagonal mass matrices $\underline{B}$ and $\underline{\tilde{B}}$ associated with the staggered mesh. This fact makes the preconditioning in (34) trivial.

We now proceed with the investigation of the conditioning of the discrete pressure operator resulting from spectral element discretization of the semiperiodic model problem. In Maday, Patera, and Rønquist [30], it is shown that the inf-sup parameter $\beta_{h}(k)$ associated with a particular wave number $k$ is independent of the discretization $h=(K, N)$; see also [42] for another proof of this point. As long as the condition number $\kappa^{S}$ is of order unity, this result is optimal with regard to both the accuracy of the discrete pressure and the efficiency by which the pressure can be computed. We now present numerical results demonstrating the good conditioning of the preconditioned pressure matrix $\underline{\tilde{B}}^{-1} \underline{S}$ for the semiperiodic problem; in what follows, $\lambda_{l}^{S}(k)$, $\kappa^{S}(k)$ will refer to the spectrum and conditioning of $\tilde{B}^{-1} \underline{S}$ for a particular wave number $k$. The calculation of the eigenvalues is based on EISPACK routines.

We begin by plotting in Fig. 1 the $\lambda_{l}^{S}(k)$ for the spectral element discretization $h=(K, N)=(4,7)$ and wave number $k=1$. The agreement with the continuous operator spectrum is seen to be virtually exact. In Fig. 2 we again plot $\lambda_{l}^{S}(k)$ with $h=(K, N)=$ $(4,7)$, but now for a wave number $k=12$. The low modes of the system are again in good agreement with the continuous spectrum. However, at this large value of $k$, the discrete system can no longer resolve exactly the higher modes, resulting in a cluster of eigenvalues at $\lambda_{*}^{S} \sim 1.2$. If we investigate the spectrum for $k=12$, but now using a discretization $h=(K, N)=(4,14)$, we see in Fig. 3 that the cluster of numerical eigenvalues has almost disappeared due to the higher spatial resolution in $x$.

In Fig. 4 we plot $\kappa^{s}$ and $\kappa^{s}$ as a function of $k$ for the spectral element and continuous operators, respectively. For small and moderate $k$ the two curves coincide; however, as $k \Rightarrow \infty$ the resolution becomes too low and the two curves diverge. For finer resolutions (e.g., larger $N$ ) the spectral element and the theoretical results agree over a larger range of wave numbers, as expected from Figs. 2 and 3. For large wave numbers $k$, the condition number $\kappa^{S}(k)$ for the spectral element discretization is larger than the value predicted by the continuous analysis, however, the value is still of order unity, as required for fast convergence of the outer iteration. 


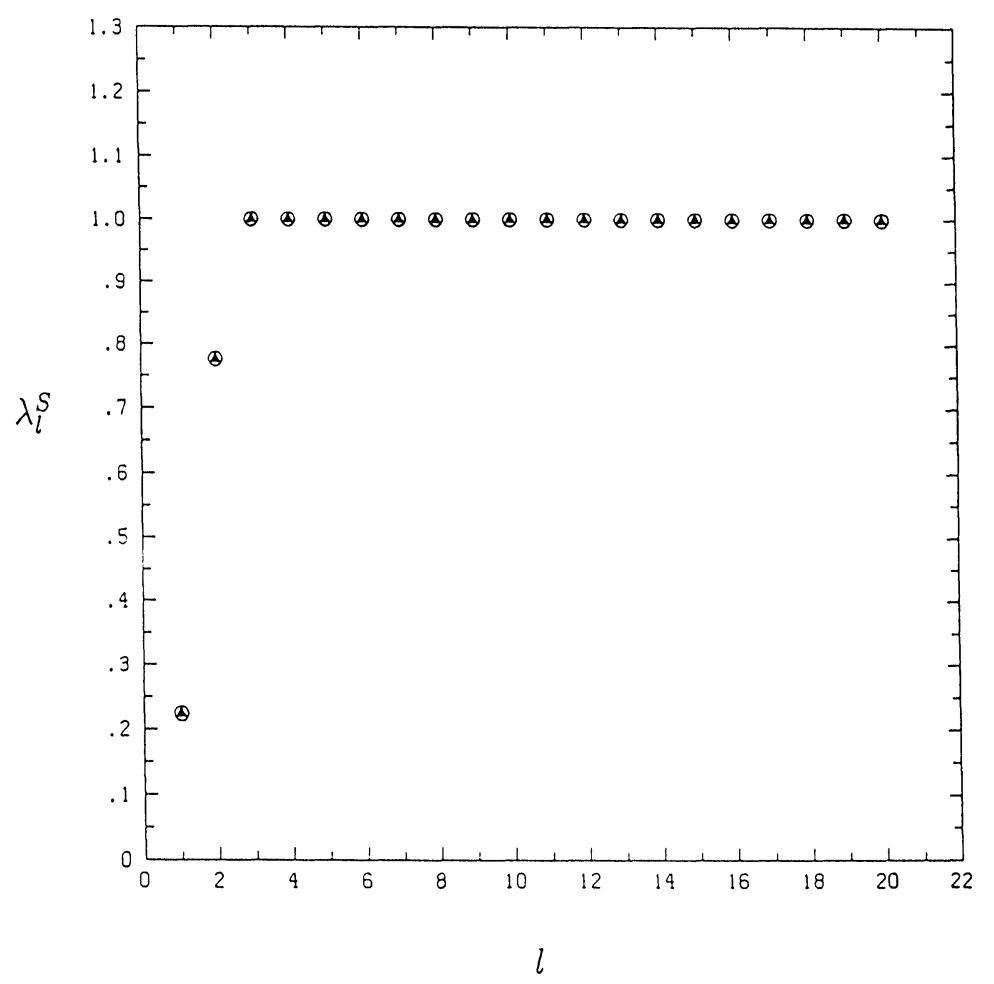

FIG. 1. A plot of the spectrum $\lambda_{l}^{S}(k)$ of the preconditioned steady Stokes pressure matrix $\underline{\tilde{B}}^{-1} \underline{S}$, where $\underline{S}$ is the pressure matrix given in (31) and $\tilde{B}$ is the mass matrix defined on the Gauss pressure mesh. The spectrum ( $\Delta)$ corresponds to a spectral element discretization $(K=4, N=7)$ for a wave number $k=1$; the agreement with the continuous operator spectrum $\lambda_{l}^{s}$ of (73)-(75) (O) is very good.

3.4. Multidimensional spectral element case. Before we present any numerical results, we make some general remarks regarding iterative solvers. First, one major reason for using iterative solvers is to avoid the severe memory requirements associated with direct methods, especially for large multidimensional problems. The computational complexity associated with an iterative solver is essentially determined by two factors: the convergence rate of the method, and the operation count for a typical matrix-vector product evaluation. In this section we focus mostly on the conditioning of the Uzawa operator $\underline{S}$, which is directly related to the convergence rate of the outer pressure iteration (34). However, we should point out that in the context of high-order methods, fast matrix-vector product evaluations are typically effected by a combination of tensor-product forms and vectorization.

The spectral element discretization procedure for the general multidimensional case is essentially a tensor-product extension of the (one-dimensional) procedure described in $\S 3.3$. In summary, the key points are the use of variational projection operators, piecewise high-order polynomial subspaces, and tensor-product bases and quadratures, resulting in minimal interelemental couplings and efficient matrix-vector product evaluations.

We consider now the Uzawa decoupling procedure (29), (30) as applied to multidimensional spectral element approximations. As discussed earlier, the pressure $\underline{p}$ is first computed from (30) by effecting a nested inner/outer iteration procedure, while (29) is solved for the velocity $\underline{u}_{i}, i=1, \ldots, d$, with known pressure $\underline{p}$. The number 


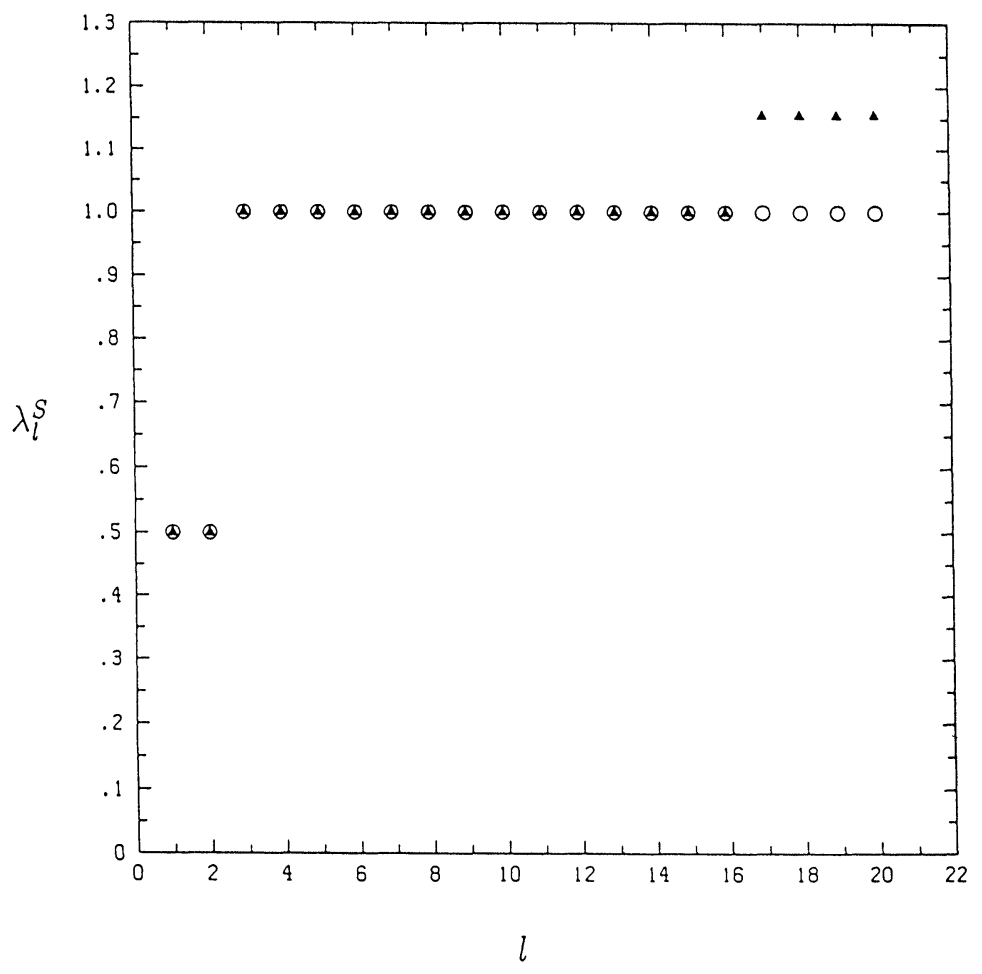

FIG. 2. A plot of the spectrum $\lambda_{l}^{S}(k)$ of the preconditioned pressure matrix $\tilde{B}^{-1} \underline{S}$. The spectrum $(\mathbf{\Delta})$ corresponds to a spectral element discretization $(K=4, N=7)$ for a wave number $k=12$; for this large value of $k$ the discrete system can no longer resolve the higher continuous modes (O).

of outer conjugate gradient iterations in (34) critically depends on the condition number $\kappa^{S}$, which we now investigate for multidimensional problems with Dirichlet velocity boundary conditions.

In order to find the condition number $\kappa^{S}$, we must compute the minimum and maximum eigenvalues of the matrix $\underline{S}$ with respect to the mass matrix $\underline{\tilde{B}}$. Since we never form any global system matrix explicitly, standard routines for calculating eigenvalues cannot be used. Instead, we compute the maximum eigenvalue $\lambda_{\max }^{S}$ using the ordinary power method [24], which involves the evaluation of matrix-vector products of the form $\underline{S} \phi$. To compute the minimum eigenvalue $\lambda_{\min }^{S}$ we use the inverse power method [24], which requires inverting the matrix $\underline{S}$ for each iteration. Note that in order to do this inversion, we use the inner/outer iteration procedure described in (34)-(37).

We start by considering the solution to the steady Stokes equations (1), (2) on a square domain $\Omega=]-1,1\left[{ }^{2}\right.$ with homogeneous Dirichlet velocity boundary conditions (3). Using a spectral element discretization with one single element, i.e., $K=1$, we compute the minimum eigenvalue $\lambda_{\min }^{S}$ of the discrete steady Stokes pressure-operator $\underline{S}$ for different values of the polynomial degree $N$. As we can see from Fig. $5, \lambda_{\text {min }}^{S}$ decreases as $N$ increases, implying that the $\beta_{h}$ in (41) is no longer independent of the discretization $h=(K, N)$. This is, in fact, numerical evidence of the presence of weak spurious modes [42]. Theoretically [8], the results indicate that $\beta_{h} \sim \mathscr{O}\left(N^{-1 / 2}\right)$ as $N \rightarrow \infty$, and hence the number of outer conjugate gradient iterations would, at worst, scale like $N^{1 / 2}$. Note that the numerical results of Fig. 5 show that the theoretical 


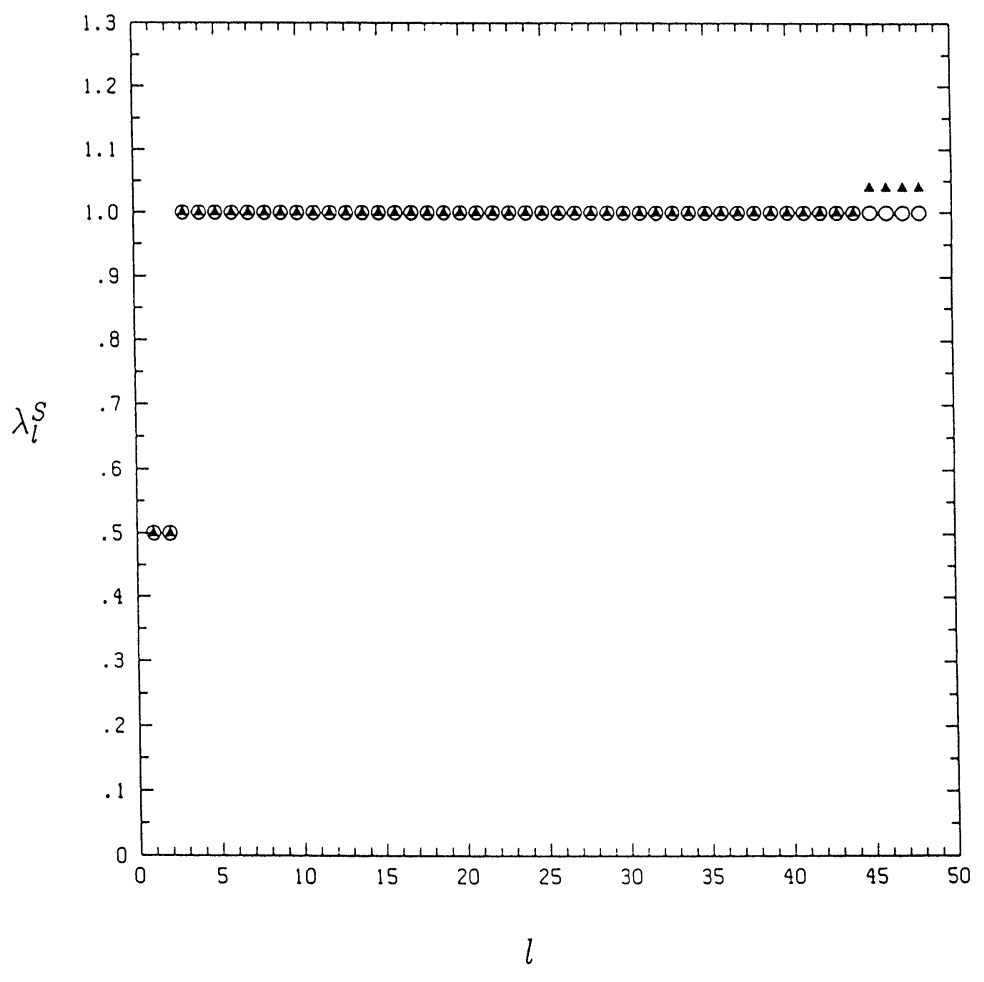

FIG. 3. A plot of the spectrum $\lambda_{l}^{S}(k)$ of the preconditioned pressure matrix $\tilde{B}^{-1} \underline{S}$. The spectrum $(\mathbf{\Delta})$ corresponds to a spectral element discretization $(K=4, N=14)$ for a wave number $k=12$; due to the higher spatial resolution the agreement of the discrete spectrum with the continuous operator spectrum $(O)$ is improved.

result is pessimistic for the low values of $N$ of interest in the spectral element context. Next, to see the effect of breaking up the domain $\Omega$ into several subdomains, we compare in Fig. 6 the minimum eigenvalue $\lambda_{\min }^{S}$ when using one single spectral element $K=1$, and when using $K=4$ and $K=16$ equal spectral elements. The results clearly show that $\beta_{h}$ is very insensitive to $K$, especially for larger $N$.

In Appendix $A$ it is shown that the maximum eigenvalue $\lambda_{\max }^{S}$ is bounded from above, and that this bound is of order unity. To demonstrate numerically that this is indeed the case, in Fig. 7 we plot $\lambda_{\max }^{S}$ for different values of the polynomial degree $N$. The results show that the maximum eigenvalue is insensitive to the number of elements $K$ and to asymptotes to a value below two, as $N$ increases. In practice, the polynomial degree $N$ is typically taken to be of order ten, suggesting that the outer pressure iteration will converge in order-one iterations. Our experience from solving a large variety of two-dimensional and three-dimensional problems is that about ten outer iterations suffice in most cases. We refer to Maday, Patera, and Rønquist [32] for theoretical proofs of the previous numerical evidence.

We now consider a two-dimensional steady Stokes test problem where preconditioned conjugate gradient iteration in the outer pressure iteration is combined with spectral element multigrid for the inner Laplacian solves. The test problem is creeping flow in a "wedge," but with the tip of the wedge removed. The spectral element discretization $(K=40, N=8)$ is shown in Fig. 8(a), and the solution in the form of streamlines is shown in Fig. 8(b). In this test problem, we have removed the tip of the 


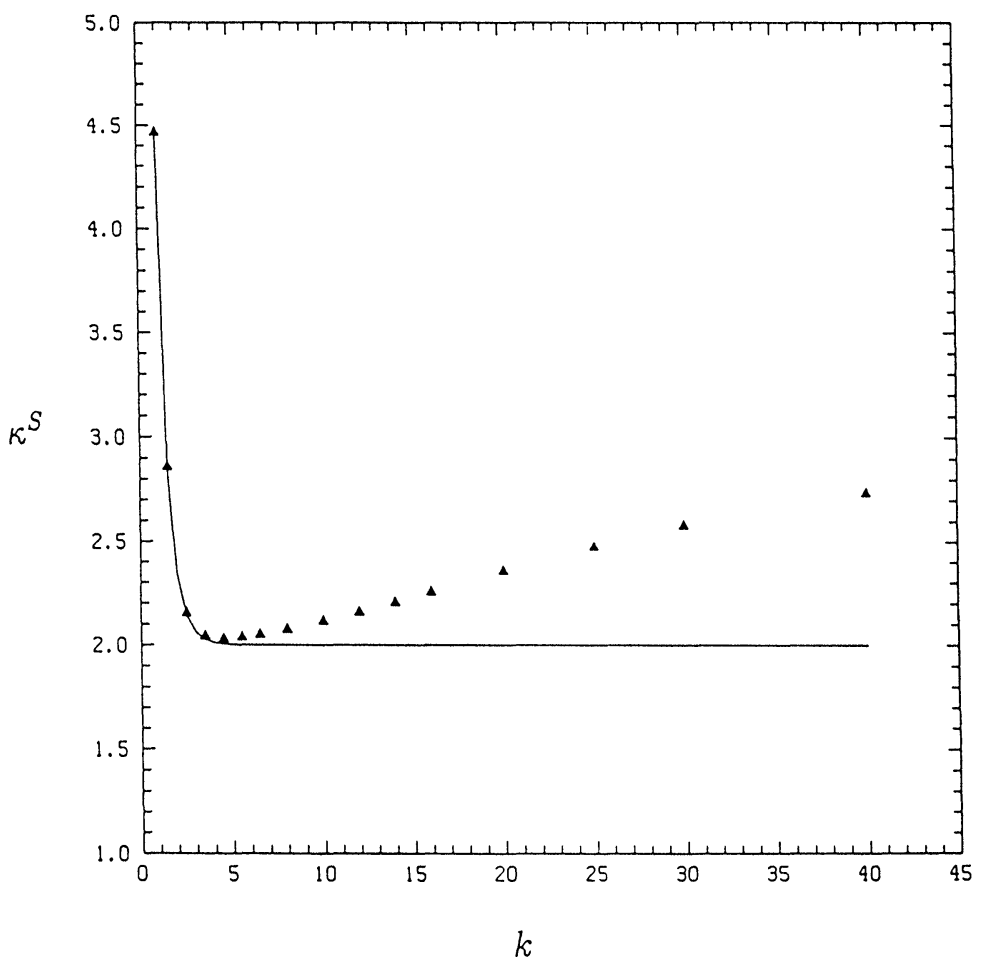

FIG. 4. A comparison between the condition number $\kappa^{s}(k)$ of the spectral element operator $\tilde{B}^{-1} \underline{S}(\mathbf{\Delta})$ and the condition number $\kappa^{s}(k)$ of the continuous operator $s$ in (65) (solid line), as a function of Fourier wave number $k$. The spectral element discretization $(K=4, N=10)$ is used. For small and moderate $k$ the two curves coincide. However, as $k \Rightarrow \infty$ the two results diverge due to the finite resolution of the spectral element mesh.

wedge in order to be able to break up the computational domain into spectral elements with aspect ratio approximately equal to unity (see Fig. 8(a)). As discussed in Ronquist [37], the convergence rate of the spectral element multigrid algorithm deteriorates as the aspect ratio of the elements becomes much different from unity. For this particular steady Stokes test problem the total speedup was about 2.5 using multigrid with $J=4$ meshes instead of preconditioned conjugate gradient iteration for the inner Laplacian solves (timings on a CRAY-XMP). Note that due to the more inefficient vectorization of the matrix-vector products on the coarser meshes $(j=1,2$, and 3$)$ compared to the finest mesh $(j=4)$, the computational cost on the coarser meshes $(j \neq J)$ cannot be neglected.

Next, we consider the Uzawa decoupling procedure as applied to a threedimensional steady Stokes problem (1), (2) in a domain $\Omega$ defined by $\left.x_{1} \in\right] 0,2 \Gamma\left[, x_{2} \in\right.$ ]$-1,1\left[, x_{3}=\right]-1,1[$, where $\Gamma$ can be interpreted as the aspect ratio of the system. The prescribed force $f$ is such that the exact solution is given by $\mathbf{u}=\left(u_{1}, u_{2}, u_{3}\right)=$ $\left(\left(1-x_{2}^{2}\right)\left(1-x_{3}^{2}\right), 0,0\right)$ and $p=\sin \pi x_{1} / \Gamma \cdot \cos \pi x_{2} \cdot \cos \pi x_{3}$. For large three-dimensional problems it is a nontrivial task to compute the eigenvalues of the pressure operator $\underline{S}$, and we therefore instead produce convergence histories from which appropriate condition numbers can be inferred. In particular, we shall plot the residual $\|\underline{r}\|_{0, h}$ (essentially the root mean square of the divergence) as a function of the number of iterations $m$ in the outer conjugate gradient iteration (34). 


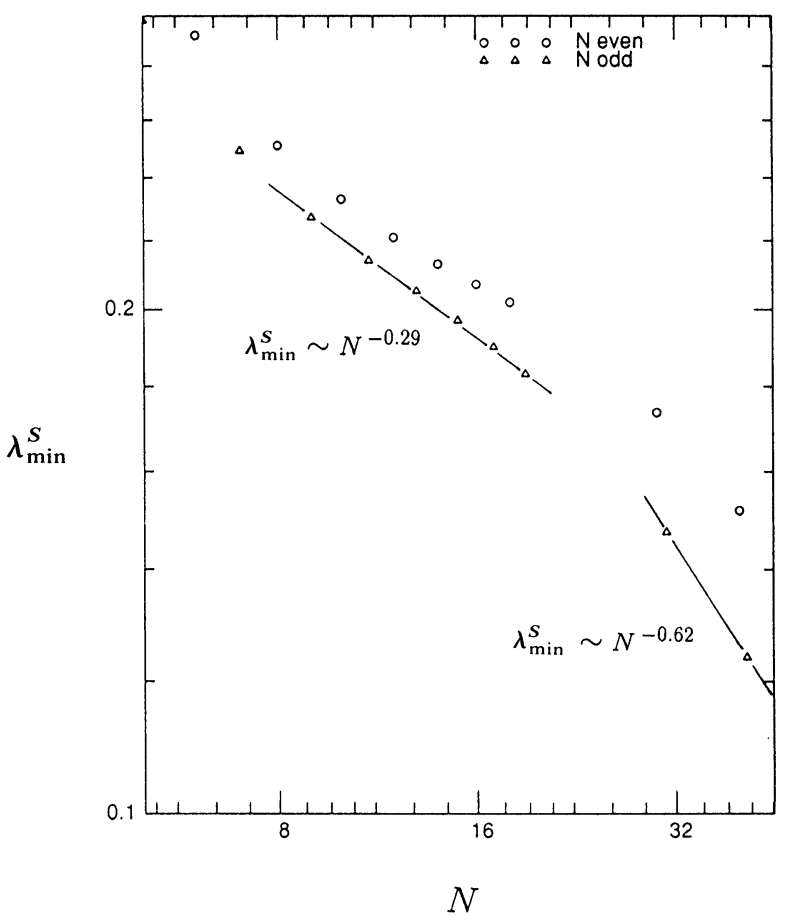

FIG. 5. A log-log plot of the minimum eigenvalue $\lambda_{\min }^{S}$ of the discrete Stokes operator $\tilde{B}^{-1} \underline{S}$ as a function of the polynomial degree $N(\triangle, N$ odd; $\bigcirc, N$ even $)$. The steady Stokes equations are solved on a square domain $\Omega=]-1,1\left[{ }^{2}\right.$ with homogeneous Dirichlet velocity boundary conditions using $K=1$ spectral elements. For low values of $N, \lambda_{\min }^{S} \sim N^{-0.29}$, implying that $\beta_{h} \sim N^{-0.15}$. For larger values of $N, \lambda_{\min }^{S} \sim N^{-0.62}$, implying that $\beta_{h} \sim N^{-0.31}$. Theoretically (Bernardi and Maday [8]), $\beta_{h}$ should at worst scale like $N^{-1 / 2}$, suggesting that the theoretical result is somewhat pessimistic for low values of $N$.

In Fig. 9 we plot $\|\underline{r}\|_{0, h}$ as a function of $m$ for an aspect ratio $\Gamma=1$ and for spectral element discretizations corresponding to $K=8, N=7$ and 10 . The initial convergence rate is almost independent of $N$, however, the asymptotic convergence rate does appear to be a weak function of $N$, in good agreement with the above discussion. In Fig. 10 we repeat the numerical experiment of Fig. 9, but now keeping the discretization parameter $h=(K, N)=(8,10)$ fixed while varying the aspect ratio $\Gamma$. The convergence rate is somewhat lower for $\Gamma=3$ as compared to $\Gamma=1$, however, the effect is small. These results demonstrate that the good conditioning of the quasi-two-dimensional (semiperiodic) model problem does, indeed, extend to multidimensional problems.

To show the potential of the Uzawa algorithm we present results from a large three-dimensional problem with a complicated geometry. The problem we consider is solving the steady Stokes equations (1), (2) in a spiral-grooved bearing with 16 grooves. Although periodicity conditions could have been exploited, the full three-dimensional problem was discretized using 312,000 degrees of freedom. The set of algebraic equations (29), (30) was then solved on a 64-processor Intel Hypercube in about 16 minutes (160 MFLOPS). The convergence history for the outer pressure iteration (34) is plotted in Fig. 11. We see that the discrete divergence is reduced by three orders of magnitude in about 30 outer iterations. Thus we have demonstrated that the Uzawa algorithm works well for large realistic problems, and can successfully be implemented on a parallel computer. For a more detailed discussion of the parallel aspects of the algorithm, we refer to Fischer and Patera [18]. 


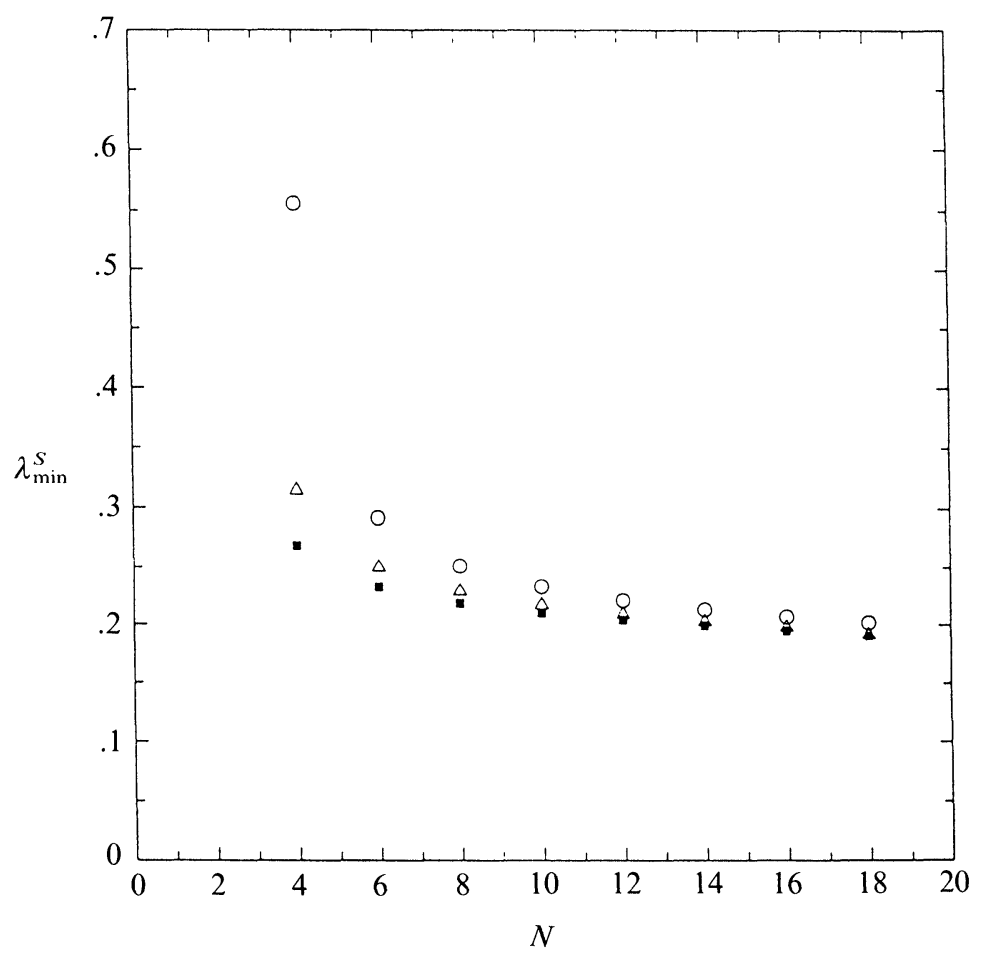

FIG. 6. A plot of the minimum eigenvalue $\lambda_{\min }^{S}$ of the discrete Stokes operator $\tilde{B}^{-1} \underline{S}$, as a function of the polynomial degree $N$. The steady Stokes equations are solved on a square domain $\Omega=]-1,1{ }^{2}$ with homogeneous Dirichlet velocity boundary conditions using $K=1(\bigcirc), K=4(\triangle)$, and $K=16(\square)$ spectral elements.

4. Unsteady Stokes solvers. In $\S 2.2$ we derived a set of algebraic equations (18), (19) resulting from a spectral element discretization of the implicitly treated unsteady Stokes problem (12)-(15). In order to compute the nodal values $\underline{\underline{u}}^{n+1}$ and $\underline{p}^{n+1}$, a classical Uzawa scheme can again be constructed, but now with the discrete Laplace operator $\underline{A}$ in (25) replaced by the discrete Helmholtz operator

$$
\underline{H}=\mu \underline{A}+\frac{\rho}{\Delta t} \underline{B} .
$$

As in the steady case, the simple gradient method can be accelerated by using conjugate gradient iteration. However, in the unsteady case we must generally consider preconditioners other than the mass matrix $\tilde{B}$ [28], [19], [38], [37], [14].

For reasons of efficiency and rigor (no rediscretization), our approach to solving the system (18), (19) will again be based on a global iterative technique. Proceeding in the same fashion as for the steady Stokes case, we arrive at the following decoupled system equivalent to the saddle problem (18), (19)

$$
\begin{aligned}
& \underline{H} \underline{u}_{i}^{n+1}=\underline{D}_{i}^{T} \underline{p}^{n+1}+\underline{g}_{i}^{n}, \quad i=1, \ldots, d, \\
& \underline{S}_{t} \underline{p}^{n+1}=-\underline{D}_{i} \underline{H}^{-1} \underline{g}_{i}^{n},
\end{aligned}
$$

where

$$
\underline{S}_{t}=\underline{D}_{i} \underline{H}^{-1} \underline{D}_{i}^{T}
$$




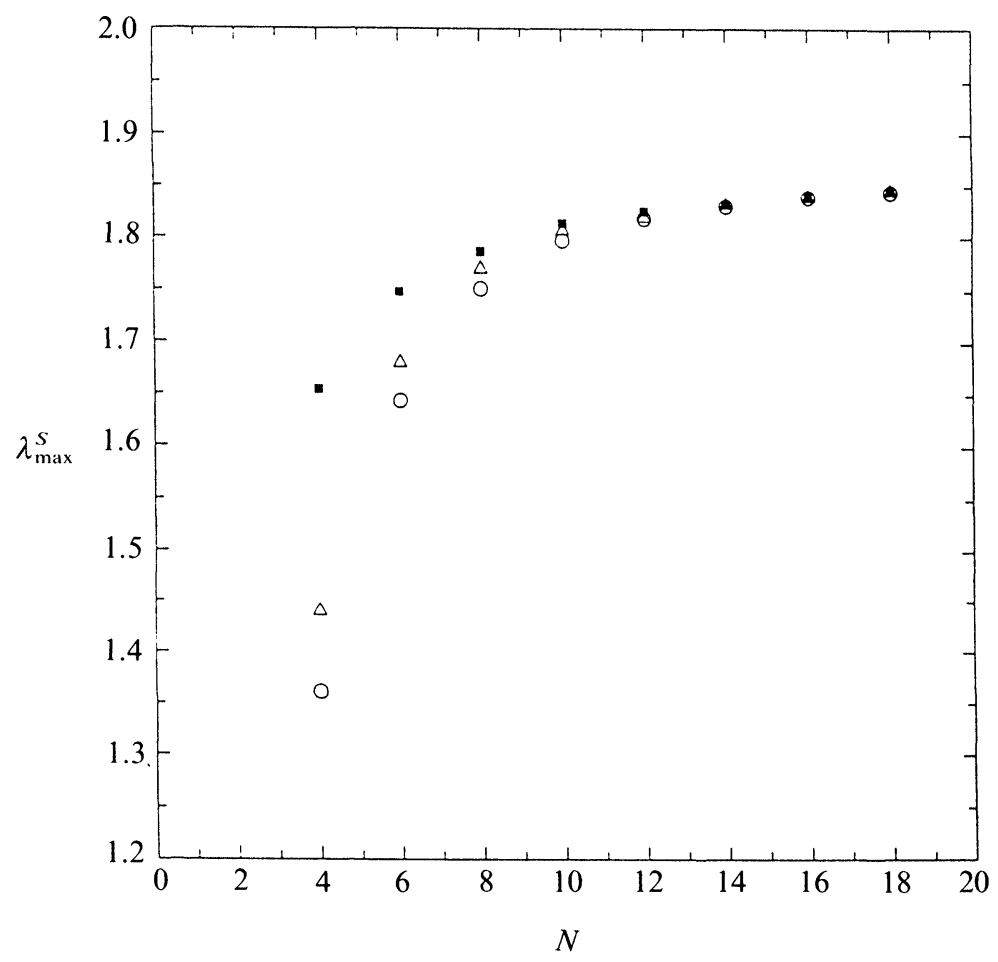

FIG. 7. A plot of the maximum eigenvalue $\lambda_{\max }^{S}$ of the discrete Stokes operator $\tilde{B}^{-1} \underline{S}$ as a function of the polynomial degree $N$. The steady Stokes equations are solved on a square domain $\Omega=]-1,1\left[{ }^{2}\right.$ with homogeneous Dirichlet velocity boundary conditions using $K=1(\bigcirc), K=4(\triangle)$, and $K=16(\square)$ spectral elements.

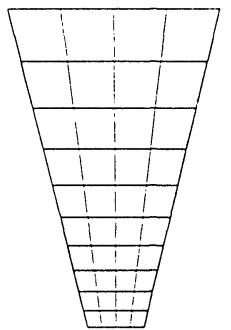

(a)

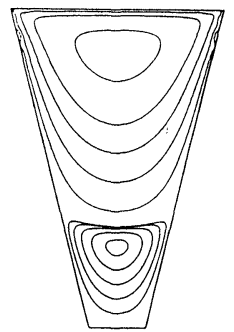

(b)

FIG. 8. Creeping flow in a "wedge" where the tip of the wedge is removed. The imposed velocity boundary conditions are nonslip conditions on the two side walls and at the bottom, with a unit horizontal velocity imposed on the top side; (a) shows the spectral element discretization $(K=40, N=8)$, while (b) shows the solution in form of streamlines.

is the unsteady Stokes pressure-operator analogous to the steady operator $\underline{S}$ defined in (31), and

$$
\underline{g}_{i}^{n}=\underline{B}\left(\underline{f}_{i}^{n}+\frac{\rho}{\Delta t} \underline{u}_{i}^{n}\right), \quad i=1, \ldots, d
$$

represent the inhomogeneities associated with an implicit Euler backward time integration procedure. The advantages of the formulation (83), (84) are similar to those for 


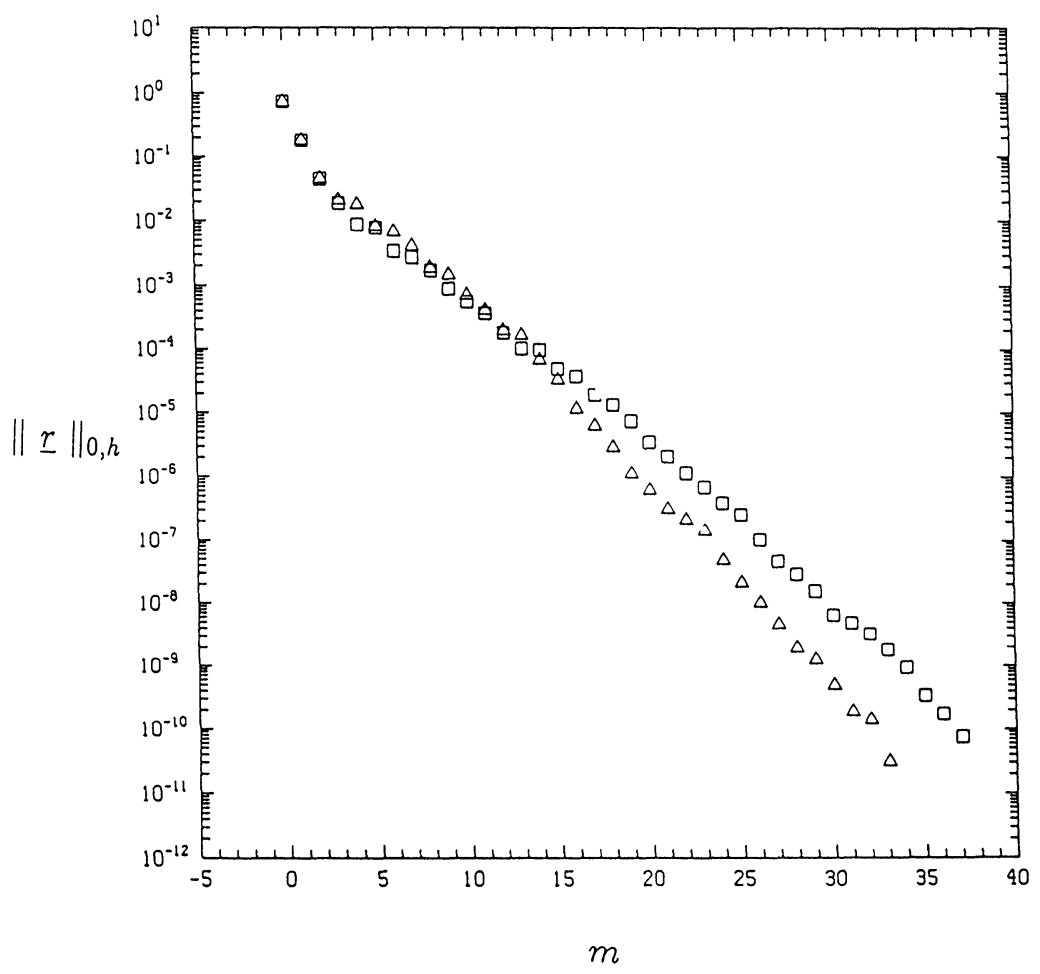

FIG. 9. A plot of the residual $\|\underline{r}\|_{0, h}$ (the root-mean-square of the divergence) from (34) as a function of the number of outer conjugate gradient iterations $m$ when solving the three-dimensional steady Stokes problem with solution $\mathbf{u}=\left[\left(1-x_{2}^{2}\right)\left(1-x_{3}^{2}\right), 0,0\right], p=\sin \pi x_{1} / \Gamma \cdot \cos \pi x_{2} \cdot \cos \pi x_{3}$ on the domain $\left.\Omega=\right] 0,2 \Gamma[\times]-1$, $1[\times]-1,1[$ with $\Gamma=1$. The domain is broken up into $K=8$ equal spectral elements, with convergence histories shown for $N=7(\triangle)$ and $N=10(\square)$. The convergence rate decreases slightly with increasing $N$.

the steady problem; it represents a complete, general, velocity-pressure decoupling that is discretely equivalent to the original discretization (18), (19). First, we solve (84) for the pressure, and then (83) is solved for each velocity component $\underline{u}_{i}^{n+1}$ with $\underline{p}^{n+1}$ known.

As for the steady Stokes problem the matrix $S_{t}$ is completely full, and therefore solving (84) requires an iterative approach. Unfortunately, whereas the steady pressureoperator $\underline{S}$ is naturally well conditioned $\left(\tilde{B}^{-1} \underline{S}\right.$ is close to the identity), the same is not true for $\underline{S}_{t}$. For large time steps we can express $\underline{S}_{t}$ as

$$
\Delta t \Rightarrow \infty, \quad \underline{S}_{t} \Rightarrow \frac{1}{\mu} \underline{S},
$$

and it is thus well conditioned. However, for small time steps, $\underline{S}_{t}$ goes to the pseudoLaplacian $\underline{E}$,

$$
\Delta t \Rightarrow 0, \quad S_{t} \Rightarrow \frac{\Delta t}{\rho} E
$$

where

$$
\underline{E}=\underline{D}_{i} \underline{B}^{-1} \underline{D}_{i}^{T}
$$




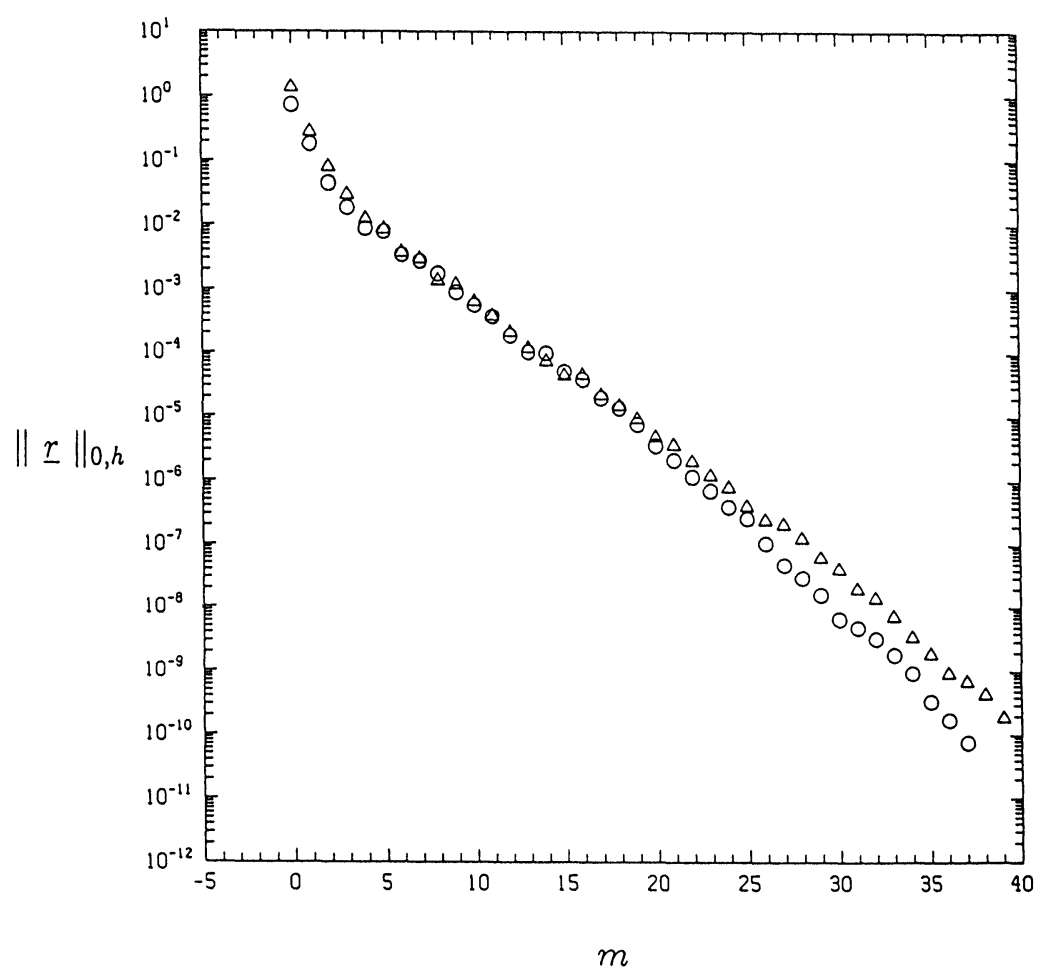

FIG. 10. A plot of the residual $\|\underline{r}\|_{0, h}$ (the root-mean-square of the divergence) from (34) as a function of the number of outer conjugate gradient iterations $m$ when solving the three-dimensional steady Stokes problem with solution $\mathrm{u}=\left[\left(1-x_{2}^{2}\right)\left(1-x_{3}^{2}\right), 0,0\right], p=\sin \pi x_{1} / \Gamma \cdot \sin \pi x_{2} \cdot \cos \pi x_{3}$ on the domain $\left.\Omega=\right]-1,1[\times]-1$, $1[\times] 0,2 \Gamma[$ with $\Gamma=1(\bigcirc)$ and $\Gamma=3(\triangle)$. Both domains are broken up into $K=8$ equal spectral elements, each of order $N=10$. The convergence rate decreases slightly as the aspect ratio $\Gamma$ increases.

is poorly conditioned. The matrix $E$ is, in fact, the discrete consistent Poisson-operator resulting from spectral element discretization of the explicitly treated unsteady Stokes problem (12)-(15). The algorithm described for the steady case therefore needs to be modified.

Earlier spectral element solvers used a two-level Richardson inner/outer iteration scheme to solve the discrete unsteady Stokes and Navier-Stokes equations [38], [31]. Computational tests indicate that the approach of Cahouet and Chabard [13] is simpler and more efficient, and we shall therefore precondition the unsteady pressure-operator $\underline{S}_{t}$ directly. The preconditioner proposed is [14]

$$
\underline{P}^{-1}=\mu \underline{\tilde{B}}^{-1}+\frac{\rho}{\Delta t} \underline{E}^{-1},
$$

which can be motivated by analyzing the two limits of very small and very large time steps. In both of these cases we expect $\underline{P}^{-1} \underline{S}_{t}$ to be close to the identity operator. As discussed in Cahouet and Chabard [14], the particular choice (90) as a preconditioner for $\underline{S}_{t}$ can perhaps be better motivated by considering the Fourier discretization (48), (49) in $\mathscr{R}^{d}$.

4.1. Multidimensional spectral element case. Our approach to inverting the unsteady pressure-operator is the same as for the steady case, namely, a nested global 


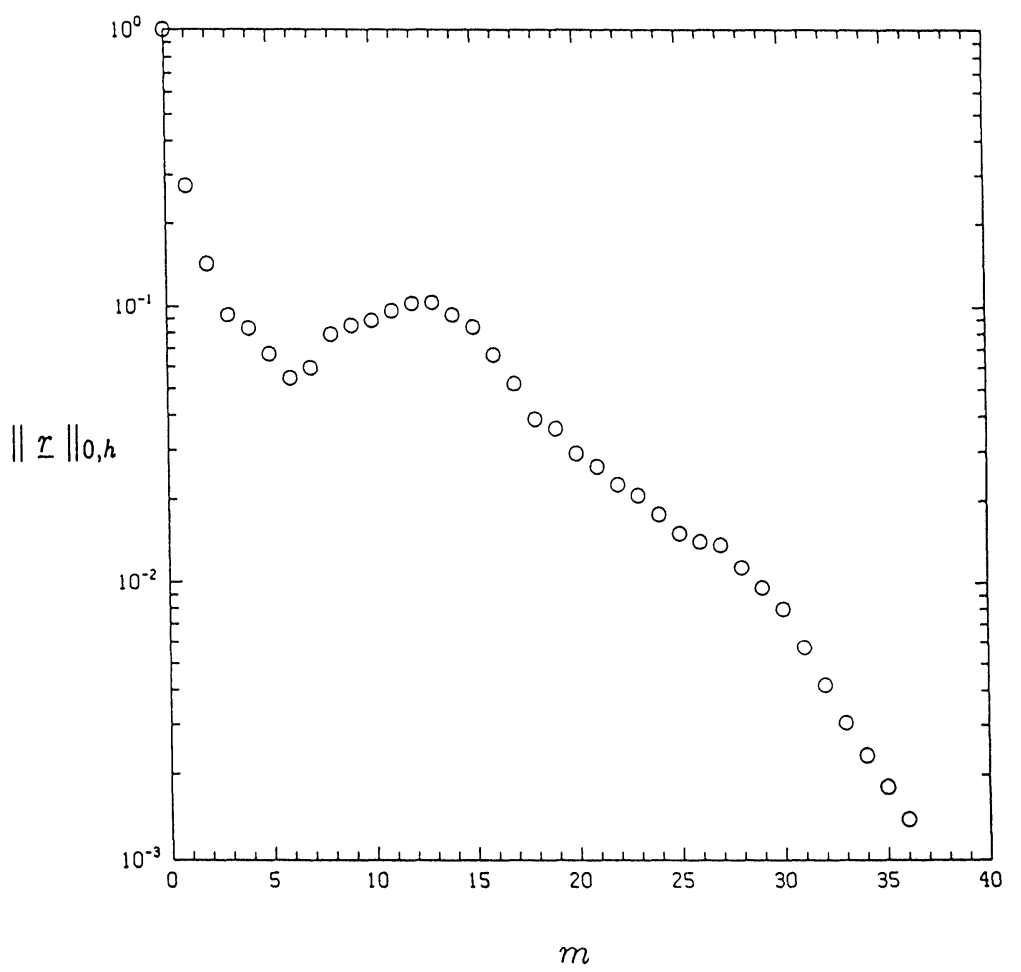

FIG. 11. A plot of the residual $\|\underline{r}\|_{0, h}$ (the root-mean-square of the divergence) from (34) as a function of the number of outer conjugate gradient iterations $m$ when solving the three-dimensional steady Stokes equations in a spiral-grooved bearing with 16 grooves. The 312,000 degrees-of-freedom problem was solved on a 64-processor Intel hypercube iPSC/2-VX in about 16 minutes at an average speed of 160 MFLOPS.

inner/outer iterative procedure based on preconditioned conjugate gradient iteration for the outer iteration, and spectral element multigrid for the inversion of the discrete Helmholtz operator $\underline{H}$. We note that the structure in the solution procedure is similar to the steady case, however, the computational complexity associated with the preconditioning in the outer iteration is very different. For the steady case the inversion of the diagonal mass matrix $\underline{B}$ is trivial, whereas the unsteady case requires the inversion of the pseudo-Laplacian $E$. If we count the inversion of the $E$-matrix as one standard elliptic solve, each iteration in the outer conjugate gradient iteration takes $d+1$ standard elliptic solves, as compared to $d$ for the steady case. If the condition number of the matrix $\underline{P}^{-1} \underline{S}_{t}$ is order unity, we see that computing the pressure again requires only order- $d$ elliptic solves. Once the pressure is known, another $d$ elliptic solves is required to compute the velocity.

We now make some remarks regarding the $E$-matrix, which is essentially a second-order operator with Neumann-like (pressure) boundary conditions. Our experience from numerical simulations has been that inverting $E$ requires more iterations than inverting the standard Laplace operator $\underline{A}$ or Helmholtz operator $\underline{H}$ with Dirichlet (velocity) boundary conditions. The slower convergence rate is probably due to the mixed $\mathscr{L}^{2}-\mathscr{H}^{1}$ spaces in the construction of the $E$-matrix. The staggered mesh also makes it more difficult to construct a proper multigrid algorithm. To this end, standard conjugate gradient iteration has been used to invert $\underline{E}$, although a multigrid approach is in preparation. 
To demonstrate the effect of the preconditioner $\underline{P}$ in (90), we monitor the residual $\|\underline{r}\|_{0, h}$ in the outer pressure iteration during the first time step when solving a (simulated) buoyancy-driven flow in a two-dimensional square cavity. We plot in Fig. 12 the convergence history for three different time steps. The larger time step $\Delta t=1$ is of the order of the time it takes to reach steady state, and $\underline{S}_{t}$ is therefore close to $\underline{S}(\mu=1)$. As expected from the steady Stokes case, we see that about ten outer iterations suffice for convergence. The smaller time step $\Delta t=10^{-4}$, however, is much smaller than a typical time scale in the system, and $\underline{S}_{t}$ is close to the pseudo-Laplacian $E$. In fact, the time step is small enough for an explicit time-stepping procedure to be stable, and we see that convergence is reached in order-one iterations. In the limit as the time step $\Delta t \Rightarrow 0$ the unsteady pressure-operator becomes perfectly preconditioned, and the steady Stokes convergence rate represents an upper bound for how fast the outer pressure iteration converges.

For comparison, we repeat in Fig. 13 the experiment of Fig. 12, but now using the preconditioner from the steady Stokes case, that is, $\underline{P}=\underline{\tilde{B}}$. As expected, as long as $\underline{S}_{t}$ is close to $\underline{S}$, the convergence rate is almost identical to the previous case. However, as the time step becomes smaller and $\underline{S}_{t}$ becomes closer to $\underline{E}$, the steady Stokes preconditioner does a poor job. In conclusion, the preconditioner (90) is an excellent preconditioner for all time steps.

We close this section by remarking that the Uzawa algorithm can readily be extended to solve the full Navier-Stokes equations by treating the nonlinear convective

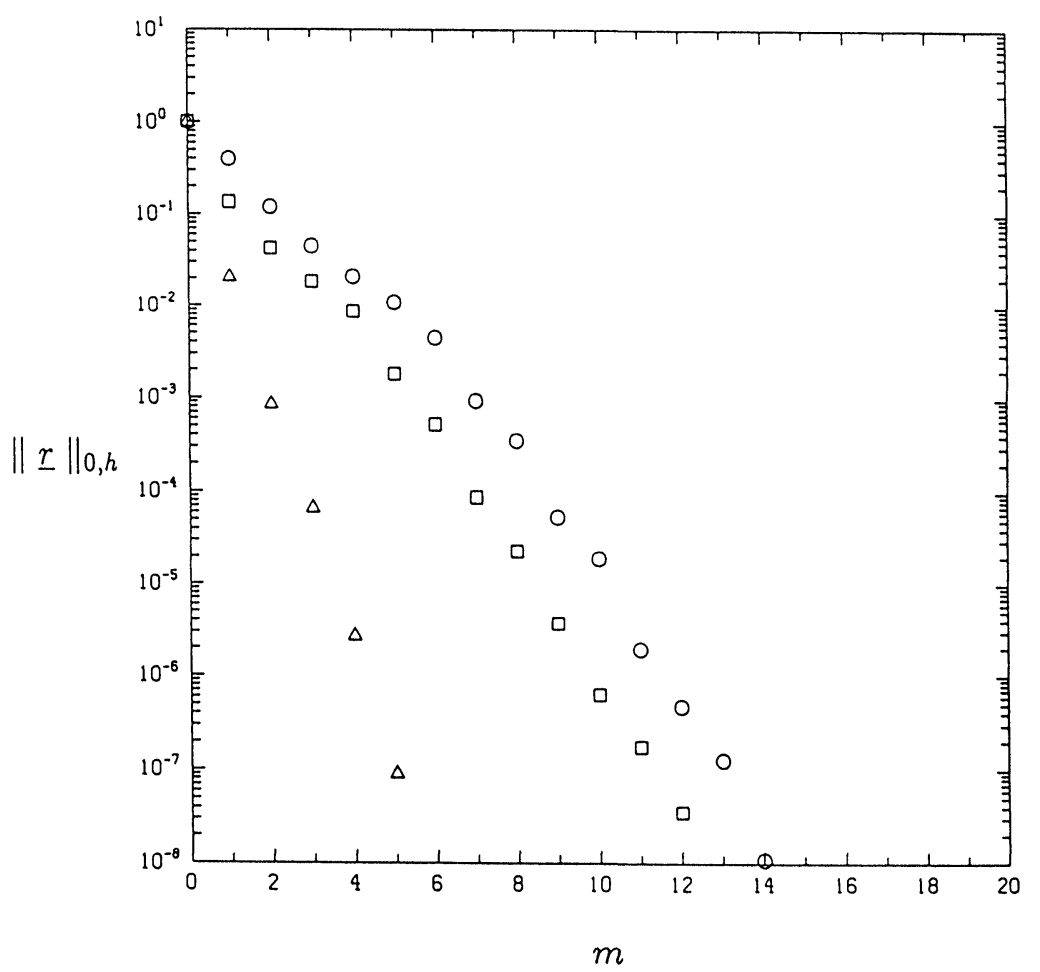

FIG. 12. A plot of the residual $\|\underline{r}\|_{0, h}$ (the root-mean-square of the divergence) as a function of the number of outer conjugate gradient iterations $m$ when solving for the first time step of a (simulated) buoyancy-driven flow in a square cavity. The plot shows the convergence history when using $\underline{P}$ defined in (90) as a preconditioner for the unsteady pressure operator $\underline{S}_{t}$ for three different time steps $\Delta t=10^{-4}(\Delta), \Delta t=10^{-2}(\square)$, and $\Delta t=1,(\bigcirc)$. 


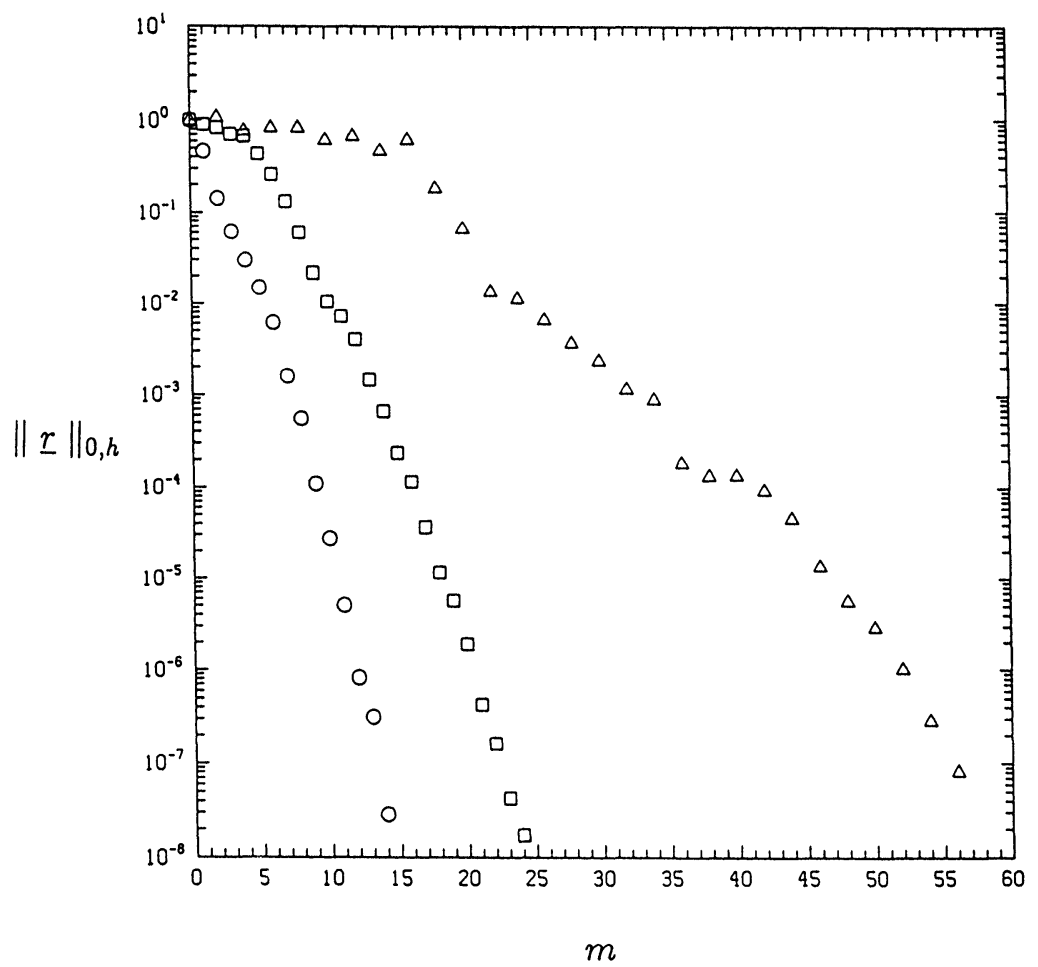

FIG. 13. A plot of the residual $\|\underline{r}\|_{0, h}$ (the root-mean-square of the divergence) as a function of the number of outer conjugate gradient iterations $m$ when solving for the first time step of a (simulated) buoyancy-driven flow in a square cavity. The plot shows the convergence history when using $\tilde{B}$ as a preconditioner for the unsteady pressure-operator $\underline{S}_{t}$ for three different time steps $\Delta t=10^{-4}(\Delta), \Delta t=10^{-2}(\square)$, and $\Delta t=1,(\bigcirc)$.

term explicitly. This approach has been used with success in the context of spectral element discretizations [17].

Appendix A. This appendix deals with relations between the condition number of the algebraic system that arises from the Uzawa algorithm and the various parameters of the discretization, in particular, the inf-sup condition, but also other constants related to the exactness of the integration formulae. To this purpose, let us recall that we have set

$$
a_{h}\left(u_{h}, v_{h}\right)=\left(\left(\nabla u_{h}, \nabla v_{h}\right)\right)_{h},
$$

and introduce the following constants

$$
\begin{aligned}
& c_{1}=\sup _{\phi_{h} \in M_{h}} \frac{\left(\phi_{h}, \phi_{h}\right)_{h}}{\left(\phi_{h}, \phi_{h}\right)}, \\
& c_{1}^{\prime}=\inf _{\phi_{h} \in M_{h}} \frac{\left(\phi_{h}, \phi_{h}\right)_{h}}{\left(\phi_{h}, \phi_{h}\right)}, \\
& \alpha=\inf _{\mathbf{v}_{h} \in X_{h}} \frac{a_{h}\left(\mathbf{v}_{h}, \mathbf{v}_{h}\right)}{\left\|\mathbf{v}_{h}\right\|_{1}^{2}} .
\end{aligned}
$$

Let us first bound the maximum eigenvalue $\lambda_{\max }^{S}$ of the matrix $\underline{S}$ with respect to the mass matrix $\tilde{\tilde{B}}$. 
To this purpose, let us first consider the discrete Laplace operator defined by $\mathbf{u}_{h}=\Delta_{h}^{-1} \mathbf{g}$ if

$$
a_{h}\left(\mathbf{u}_{h, i}, \mathbf{v}_{i}\right)=\left(\left(\mathbf{g}_{i}, \mathbf{v}_{i}\right)\right)_{h}, \quad \forall \mathbf{v} \in X_{h} .
$$

Let us now consider the operator $\operatorname{div}^{T}$, transposed by the divergence operator with respect to the $(.,)_{h}$-scalar product, i.e.,

$$
\left(\left(\operatorname{div}^{T} p, \mathbf{v}\right)\right)_{h}=(p, \operatorname{div} \mathbf{v})_{h}, \quad \forall p \in M_{h}, \quad \forall \mathbf{v} \in X_{h} .
$$

Lemma A.1. For any $p$ in $\mathscr{L}^{2}(\Omega)$, we have

$$
\left\|\Delta_{h}^{-1}(\operatorname{div})^{T} p\right\|_{1} \leqq \frac{c_{1}}{\alpha}\|p\|_{0} .
$$

Proof. This result is simply derived by taking $\mathbf{v}=\Delta_{h}^{-1}(\operatorname{div})^{T} p$ in (A.5) with $g=$ $\operatorname{div}^{T} p$, and applying the Cauchy-Schwarz inequality.

It is important to note that with these definitions, the operator $\operatorname{div}\left(\Delta_{h}^{-1}\right) \operatorname{div}^{T}$ is symmetric and that $\underline{S}$ represents its matrix in the basis of the Lagrange interpolants. Due to the symmetry, the extreme eigenvalues are related to the upper and lower norms of the images of elements of $M_{h}$ as follows:

$$
\left(\lambda_{\max }^{S}\right)=\sup _{p \in M_{h}}\left(\left[\operatorname{div}\left(\Delta_{h}^{-1}\right) \operatorname{div}^{T}\right] p, p\right)_{h}, \quad(p, p)_{h}=1
$$

and

$$
\left(\lambda_{\min }^{S}\right)=\inf _{p \in M_{h}}\left(\left[\operatorname{div}\left(\Delta_{h}^{-1}\right) \operatorname{div}^{T}\right] p, p\right)_{h}, \quad(p, p)_{h}=1 .
$$

Note that these eigenvalues are also those of the matrix $\underline{S}$ with respect to the mass matrix $\underline{\tilde{B}}$. From (A.2), (A.3), and Lemma A.1, it is an easy matter to check that

$$
\lambda_{\max }^{S} \leqq \frac{c_{1}^{2}}{\alpha c_{1}^{\prime}} .
$$

Let us now consider $\lambda_{\min }^{S}$. We want to get the relationship between this eigenvalue and the inf-sup condition constant $\beta_{h}$ given by

$$
\beta_{h}=\inf _{p \in M_{h}} \sup _{\mathbf{v} \in X_{h}} \frac{(p, \operatorname{div} \mathbf{v})_{h}}{\left(\left(\nabla \mathbf{v}_{i}, \nabla \mathbf{v}_{i}\right)\right)_{h}^{1 / 2}}, \quad(p, p)_{h}=1 .
$$

Let $p$ be given in $M_{h}$ with $(p, p)_{h}=1$. As noted by Vandeven [42, V, Théorème II.1], the elements $\mathbf{u}^{*}$ that realize the supremum related to the inf-sup condition, i.e.,

$$
\sup _{\mathbf{v} \in X_{h}} \frac{(p, \operatorname{div} \mathbf{v})_{h}}{\left(\left(\nabla \mathbf{v}_{i}, \nabla \mathbf{v}_{i}\right)\right)_{h}^{1 / 2}}=\frac{\left(p, \operatorname{div} \mathbf{u}^{*}\right)_{h}}{\left(\left(\nabla \mathbf{u}_{i}^{*}, \nabla \mathbf{u}_{i}^{*}\right)\right)_{h}^{1 / 2}}
$$

are colinear to the element $\tilde{\mathbf{u}}^{*}$ of $\boldsymbol{X}_{h}$, solution of the problem

$$
\tilde{\mathbf{u}}^{*}=\Delta_{h}^{-1} \operatorname{div}^{T} p .
$$

Since the proof of the fact is very short and simple, let us repeat it here. From (A.9) we have

$$
\frac{(p, \operatorname{div} \mathbf{v})_{h}}{\left(\left(\nabla \mathbf{v}_{i}, \nabla \mathbf{v}_{i}\right)\right)_{h}^{1 / 2}}=\frac{\left(\left(\nabla \tilde{\mathbf{u}}_{i}^{*}, \nabla \mathbf{v}_{i}\right)\right)_{h}}{\left(\left(\nabla \mathbf{v}_{i}, \nabla \mathbf{v}_{i}\right)\right)_{h}^{1 / 2}}
$$


The Cauchy-Schwarz inequality then gives the inequality

$$
\frac{(p, \operatorname{div} \mathbf{v})_{h}}{\left(\left(\nabla \mathbf{v}_{i}, \nabla \mathbf{v}_{i}\right)\right)_{h}^{1 / 2}} \leqq\left(\left(\nabla \tilde{\mathbf{u}}_{i}^{*}, \nabla \tilde{\mathbf{u}}_{i}^{*}\right)\right)_{h}^{1 / 2}
$$

with the equality if and only if $\tilde{\mathbf{u}}_{i}^{*}$ and $\mathbf{v}$ are colinear. This leads from (A.9) to

(A.10) $\sup _{\mathbf{v} \in X_{h}} \frac{(p, \operatorname{div} \mathbf{v})_{h}}{\left(\left(\nabla \mathbf{v}_{i}, \nabla \mathbf{v}_{i}\right)\right)_{h}^{1 / 2}}=\left(\operatorname{div}^{T} p,\left(\Delta_{h}^{-1}\right) \operatorname{div}^{T} p\right)_{h}^{1 / 2}=\left(\left[\operatorname{div}\left(\Delta_{h}^{-1}\right) \operatorname{div}^{T}\right] p, p\right)_{h}^{1 / 2}$,

so that the inf-sup condition satisfies

$$
\beta_{h}=\inf _{p \in M_{h}}\left(\left[\operatorname{div}\left(\Delta_{h}^{-1}\right) \operatorname{div}^{T}\right] p, p\right)_{h}^{1 / 2}, \quad(p, p)_{h}=1 .
$$

Now recalling (A.7), we have

$$
\lambda_{\min }^{S}=\beta_{h}^{2} .
$$

Finally, the condition number $\kappa^{S}$ is then bounded by $c_{1}^{2} \beta_{h}^{2} / \alpha c_{1}^{\prime}$.

Appendix B. We consider here the solution to the eigenvalue problem (71):

$$
\int_{-1}^{1}\left\{\frac{\partial}{\partial x} G\left(x, x^{\prime}\right) \frac{d \chi\left(x^{\prime}\right)}{d x^{\prime}}-k^{2} G\left(x, x^{\prime}\right) \chi\left(x^{\prime}\right)\right\} d x^{\prime}=\lambda \chi(x),
$$

where the Green's function $G\left(x, x^{\prime}\right)$ is given in closed form in (68).

To reduce the integral equation (B.1) to the form of a standard eigenvalue problem, we integrate the term involving the derivative of $\chi$ by parts,

$$
\int_{-1}^{1} \frac{\partial G}{\partial x} \frac{d \chi}{d x^{\prime}} d x^{\prime}=\left.\frac{\partial G}{\partial x} \chi\right|_{-1} ^{x^{-}}+\left.\frac{\partial G}{\partial x} \chi\right|_{x^{+}} ^{1}-\int_{-1}^{1} \frac{\partial^{2} G}{\partial x \partial x^{\prime}} \chi d x^{\prime}
$$

Here the integral is broken up into two parts due to the jump discontinuity in the derivatives of $G$,

$$
\frac{\partial G}{\partial x^{\prime}}\left(x, x^{+}\right)-\frac{\partial G}{\partial x^{\prime}}\left(x, x^{-}\right)=1 .
$$

Using (B.2) and (B.3), we can write (B.2) as

$$
\chi(x)-\int_{-1}^{1}\left\{\frac{\partial^{2} G}{\partial x \partial x^{\prime}}+k^{2} G\right\} \chi\left(x^{\prime}\right) d x^{\prime}=\lambda \chi(x),
$$

which is a homogeneous Fredholm integral equation of the second kind.

Using the explicit form of $G$ from (68) and evaluating the derivatives, we obtain

$$
\begin{aligned}
& -\int_{-1}^{1} \frac{k^{2}}{k \sinh 2 k}\left\{\cosh k\left(1+x^{<}\right) \cosh k\left(1-x^{>}\right)\right. \\
& \left.\quad+\sinh k\left(1+x^{<}\right) \sinh k\left(1-x^{<}\right)\right\} \chi\left(x^{\prime}\right) d x^{\prime} \\
& =(\lambda-1) \chi(x) .
\end{aligned}
$$

Using the definitions of $x^{<}$and $x^{>}$in (69), (70), (B.5) reduces to the following symmetric eigenvalue problem:

$$
\frac{-k}{\sinh 2 k} \int_{-1}^{1} \cosh k\left(x+x^{\prime}\right) \chi\left(x^{\prime}\right) d x^{\prime}=(\lambda-1) \chi(x) .
$$


Expanding the kernel in (B.6) we arrive at

$$
\begin{aligned}
& -\frac{k}{\sinh 2 k}\left\{\cosh k x \int_{-1}^{1} \cosh k x^{\prime} \chi\left(x^{\prime}\right) d x^{\prime}+\sinh k x \int_{-1}^{1} \sinh k x^{\prime} \chi\left(x^{\prime}\right) d x^{\prime}\right\} \\
& =(\lambda-1) \chi(x) .
\end{aligned}
$$

By inspection, it is clear that there are two solutions to (B.7) given by

$$
\begin{array}{ll}
\chi^{e}=\cosh k x, & \lambda^{e}=\frac{1}{2}-\frac{k}{\sinh 2 k}, \\
\chi^{o}=\sinh k x, & \lambda^{o}=\frac{1}{2}+\frac{k}{\sinh 2 k},
\end{array}
$$

where superscripts $e$ and $o$ denote even and odd, respectively. In addition, there exists an infinite set of eigenfunctions $\chi$ corresponding to $\lambda=1$, satisfying

$$
\int_{-1}^{1} \cosh k x^{\prime} \chi\left(x^{\prime}\right) d x^{\prime}=\int_{-1}^{1} \sinh k x^{\prime} \chi\left(x^{\prime}\right) d x^{\prime}=0 .
$$

For example, we can choose $\chi$ odd such that

$$
\int_{-1}^{1} \cosh k x^{\prime} \chi\left(x^{\prime}\right) d x^{\prime}=0
$$

or $\chi$ even such that

$$
\int_{-1}^{1} \sinh k x^{\prime} \chi\left(x^{\prime}\right) d x^{\prime}=0
$$

There are many ways to do this, which explains why the spectrum is clustered around unity.

Acknowledgments. We would like to thank Paul Fischer at Brown University, and Intel Scientific Computers for providing us with the parallel spectral element results.

\section{REFERENCES}

[1] K. Arrow, L. Hurwicz, And H. Uzawa, Studies in Nonlinear Programming, Stanford University Press, Stanford, CA, 1958.

[2] M. Azarez, G. Labrosse, and H. Vandeven, A pressure field pseudospectral evaluation for $3 D$ numerical experiments in incompressible fluid dynamics, in Proc. 11th Internat. Conf. Numerical Methods in Fluid Dynamics, D. L. Dwoyer, M. Y. Hussaini, and R. G. Voigt, eds., Springer-Verlag, Berlin, Heidelberg, New York, 1989.

[3] I. BABUŠKA, Error bounds for the finite element method, Numer. Math., 16 (1971), pp. 322-333.

[4] K. J. BATHE AND J. DONG, Solution of incompressible viscous fluid flow with heat transfer, J. Comput. Structures, to appear.

[5] C. Bernardi, C. CANUto, AND Y. MADAY, Generalized inf-sup condition for Chebyshev spectral approximation of the Stokes problem, SIAM J. Numer. Anal., 25 (1988), pp. 1237-1271.

[6] C. BERNARDI AND Y. MADAY, A collocation method over staggered grid for the Stokes problem, Internat. J. Numer. Methods Fluids, 8 (1988), pp. 537-557.

[7] - Relèvement polynomial de traces et applications, $M^{2} A N, 24$ (1990), pp. 557-611.

[8] — Approximation spectrales de problèmes aux limites elliptiques, Mathématiques et Applications, 10, Ellipse édition marketing, Springer-Verlag, Paris, 1992.

[9] C. Bernardi, Y. MAdAy, AND B. MÉTIVET, Spectral approximation of the periodic-nonperiodic Navier-Stokes equations, Numer. Math., 51 (1987), pp. 655-700. 
[10] C. Bernardi, Y. Maday, And B. MÉtivet, Calcul de la pression dans la résolution spectrale du problème de Stokes, Recherche Aerospatiale, 1987, pp. 1-21.

[11] F. BREZZI, On the existence, uniqueness and approximation of saddle-point problems arising from Lagrange multipliers, RAIRO Anal. Numer., 8 R2 (1974), pp. 129-151.

[12] M. O. Bristeau, R. Glowinski, AND J. Periaux, Numerical methods for the Navier-Stokes equations. Applications to the simulation of compressible and incompressible viscous flows, Comput. Phys. Rep., to appear.

[13] J. CAHOUET AND J. P. CHABARD, Multi-domains and multi-solvers finite element approach for the Stokes problem, Proceedings of the Fourth International Symposium on Innovative Numerical Methods in Engineering, R. P. Shaw, ed., Springer-Verlag, New York, 1986, p. 317.

[14] _ Some fast $3 d$ finite element solvers for the generalized Stokes problem, Internat. J. Numer. Methods Fluids, 8 (1988), pp. 869-895.

[15] A. J. CHORIN, Numerical solution of incompressible flow problems, in Studies in Numerical Analysis 2, J. M. Ortega and W. C. Rheinboldt, eds., Society for Industrial and Applied Mathematics, Philadelphia, PA, 1970.

[16] P. J. Davis ANd P. Rabinowitz, Methods of Numerical Integration, Academic Press, New York, 1985.

[17] P. Fischer, L. W. Ho, G. E. KARniadakis, E. M. RønQuist, AND A. T. PATERA, Recent advances in parallel spectral element simulation of unsteady incompressible flows, Comput. \& Structures, 30 (1988), pp. 217-231.

[18] P. Fischer And A. T. PATera, Parallel spectral element solution of the Stokes problem, J. Comput. Phys., 92 (1991), pp. 380-421.

[19] P. Fischer, E. Rønquist, D. Dewey, and A. T. Patera, Spectral element methods: Algorithms and architectures, in First International Symposium on Domain Decomposition Methods for Partial Differential Equations, Society for Industrial and Applied Mathematics, Philadelphia, PA, 1987, pp. 173-197.

[20] M. Fortin AND R. Glowinski, Augmented Lagrangian Methods, North-Holland, Amsterdam, 1983.

[21] V. Girault ANd P. A. Raviart, Finite Element Approximation of the Navier-Stokes Equations, Springer-Verlag, Berlin, Heidelberg, Germany, 1986.

[22] R. Glowinski, Numerical Methods for Nonlinear Variational Problems, Springer-Verlag, Berlin, New York, 1984.

[23] R. Glowinski AND O. Pironneau, On a mixed finite element approximation of the Stokes problem, Numer. Math., 33 (1979), pp. 397-424.

[24] G. H. Golub and C. F. Van Loan, Matrix Computations, The Johns Hopkins University Press, Baltimore, MD, 1983.

[25] S. JENSEN AND M. VOGELIUS, Divergence stability in connection with the p-version of the finite element methods, $M^{2} A N, 24$ (1990), pp. 737-764.

[26] J. KIM AND P. MoIN, Application of a fractional-step method to incompressible Navier-Stokes equations, J. Comput. Phys., 59 (1985), pp. 308-323.

[27] L. KLEISER AND U. SCHUMANN, Spectral simulation of the laminar turbulent transition process in plane Poiseuille flow, in Spectral Methods for Partial Differential Equations, R. G. Voigt, D. Gottlieb, and M. Y. Hussaini, eds., Society for Industrial and Applied Mathematics, Philadelphia, PA, 1984.

[28] G. LABADIE AND P. LASBleiz, Quelques métodes de résolution du problème de Stokes en éléments finis, Tech. Rep. HE41/83.01, Electricité de France, 1983.

[29] P. LEQUÉRÉ, Mono and multi domain Chebyshev algorithm on a staggered grid, in Proc. Seventh Internat. Conf. Finite Element Methods in Flow Problems, 1985.

[30] T. MADAY, A. T. PATERA, AND E. M. RøNQUIST, A well-posed optimal spectral element approximation for the Stokes problem, Tech. Rep. No. 87-48, 1987, ICASE, Hampton, VA.

[31] Y. MADAY AND A. T. PATERA, Spectral element methods for the Navier-Stokes equations, in State-of-theArt Surveys in Computational Mechanics, A. K. Noor, ed., ASME, New York, 1989, pp. 71-143.

[32] Y. MAdAy, A. T. PATERA, AND E. M. RøNQUiST, Optimal Legendre spectral element methods for the multi-dimensional Stokes problem, in preparation.

[33] Y. MAdAy AND A. QUARTERONI, Spectral and pseudospectral approximation of the Navier-Stokes equations, SIAM J. Numer. Anal., 19 (1982), pp. 761-780.

[34] J. F. MAître, F. MuSY, AND P. Nigon, A fast solver for the Stokes equations using multigrid with a Uzawa smoother, in Advances in Multi-Grid Methods, D. Braess, U. Hackbusch, and W. Trottenberg, eds., Vieweg, Braunschweig, Wiesbaden, 1985.

[35] S. A. Orszag, M. Israeli, ANd M. O. Deville, Boundary conditions for incompressible flows, J. Sci. Comput., 1 (1986), p. 75.

[36] A. T. PATERA, A spectral element method for fluid dynamics; laminar flow in a channel expansion, J. Comput. Phys., 54 (1984), pp. 468-488. 
[37] E. M. RøNQUIST, Optimal spectral element methods for the unsteady three-dimensional incompressible Navier-Stokes equations, Ph.D. thesis, Dept. of Mechanical Engineering, Massachusetts Institute of Technology, Cambridge, MA, 1988.

[38] E. M. Rønquist ANd A. T. PAtera, A Legendre spectral element method for the incompressible Navier-Stokes equations, in Proceedings of the Seventh GAMM Conference on Numerical Methods in Fluid Mechanics, Vieweg, Braunschweig, Wiesbaden, 1988.

[39] G. SACChi-LANDRIANi AND H. VANDEVEn, A multidomain spectral collocation method for the Stokes problem, Numer. Math., 58 (1990), pp. 441-464.

[40] C. STREeTt, M. Y. HuSSAINI, AND Y. MADAY, Two spectral collocation algorithms for the incompressible Navier-Stokes equations with two nonperiodic directions, in Proc. First Internat. Conf. Indust. Appl. Math., Paris, France, 1987.

[41] R. TEMAM, Navier-Stokes Equations. Theory and Numerical Analysis, North-Holland, Amsterdam, 1977.

[42] H. VANDEVEN, Compatibilité des espaces discrets pour l'approximation spectrale du problème de Stokes périodique non périodique, $M^{2} A N, 4$ (1989), pp. 649-688.

[43] - Analysis of the eigenvalues of spectral differentiation operators, manuscript.

[44] R. Verfurth, A preconditioned conjugate residual algorithm for the Stokes problem, in Multi-Grid Methods, D. Braess, W. Hackbusch, and U. Trottenberg, eds., Vieweg, Braunschweig, Wiesbaden, 1985.

[45] Y. YAMAGUCHI, C. J. CHANG, AND R. A. BROWN, Multiple buoyancy-driven flows in a vertical cylinder heated from below, Philos. Trans. Roy. Soc. London, A 312 (1984), pp. 519-552. 